

Wm NDOAC

स्थ

fuithsencian orst. oct. 1870 .

Division of Mallugetses

Sectional Library 



\section{.}


cerological Suroen of Cenlifornia.

\section{GEOGRAPHICAL CATALOGUE}

OF THE

M O L L U S C A.

Division of Mollunetsto

Sectional Iibrour? 



\title{
GEOLOGICAL SURVEY OF CALIFORNIA.
}

J. D. Winterey, State Geologist.

\author{
GEOGRAPHICAL
}

\section{CITALOGUE OF TIE MOLLUSCA}

FOUND IVEST OF THE ROCKY MOUNTAINS,

BE'TWEN LATITUDES $33^{\circ}$ AND $49^{\circ}$ NORTH.

B Y J. G. C O O P E R, M. D.

SAN FRANCISCO:

Towne \& Bacon, Printers.

तोil 1867 .

JAN 191988

4íRURES 


\section{I()TI I'L}

The following list is based on that published by 1'. P'. Carpexter, in his Report to the British Association for the Adrancement of Science, 1868 , with the addition of about 130 species which have been described since then, or were omitted. The land and fresh water species, at that time only partially determinerl and not embodied in the general list, are also added. 'The changes in range of distribution here made are founded on more recent and correct information than was at his command, including unpublished collections of Dr. Canfield, of Monterey; Dr. Neweomb, of Oakland; Rer. J. Rowell, Mr. J. Hepburn and R. E. C. Stearns, of San Francisco; Mr. Gabb, Prof. Brewer and Mr. Bolander, of the Geological Surrey; Mr. W. II. Dall, of the W. U. T'elegraph Expedition, and others, whose special discoveries and labors have been all acknowledged and tabulated in the lieport on Mollusea to be published as one of the series of the Geological Survey.

There are twenty or thirty species still undetermined, and now in Mr. Carpenter's hands, which will appear in the final report.

It need searcely be added libat this list is not intented for a final authority on the subject of names, which will probably be considerably changerl by the time the rolume on Mollusea is printed.

Most of these living species are found fossil also in the tertiary formations along this coast, and have a wider range of distribution, many extending inland to the Sierra Nevada, but the localities could not be included here. Speeimens are found nearly as perfect as the living shells.

The list is intended for use hoth as a reference or check-list, and for labels. Copies may be obtained at the office of the State Geological Survey, No. 90 Montgomery Block, San Francisco.

N. 13.-P'omzoa and 'Tuntcata are not inchuled.

San Fraxcisco, April, 1867. 

1. It tide allida, Bal, Am. I. Conch, vi, p.157 1870. Sta. Martian. News. Diego, Coop. L. Cal. Hinds. Var? Palmers, EnIf Cal. Dr. Palmer.

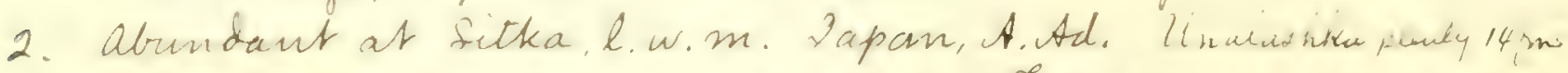

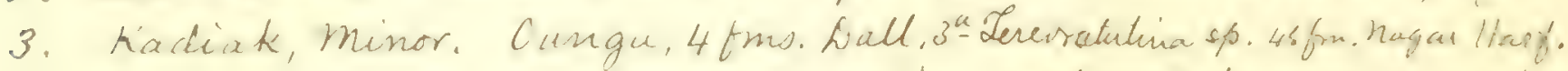

4. 2 from Orange Harbor, Patagonia, referred by an essen to Oregon in Cpr. Rep. 13. A. and Hel. Ex. Ex. It is a Teretratelle.

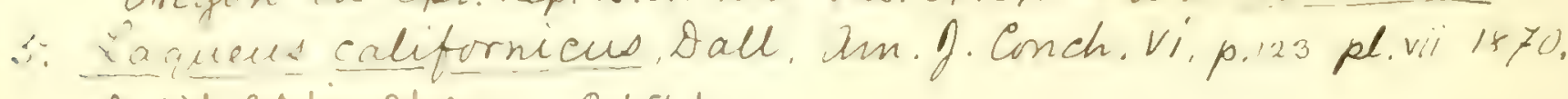
$n$. Side Catalina od Cooper. Port Etches.

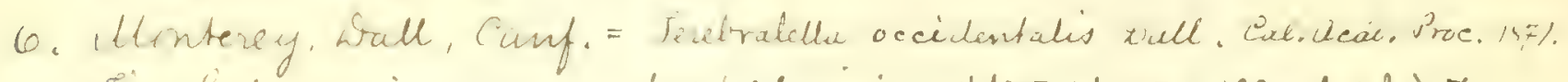

The Gala. specimens are not Wald hernias. Vol Tr. pt. Tr. p.182, pl.1.fig.7.

7. Has not been obtained from W.C.N. An.

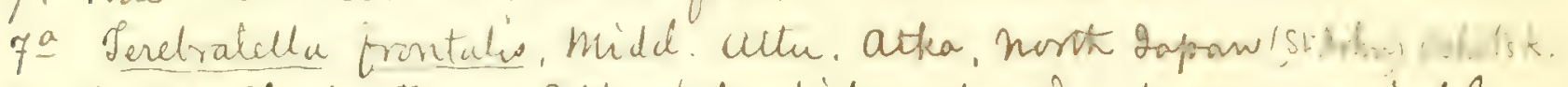

8. A true Ferebratella. Seethe, veto. tides, abundant, very variable.

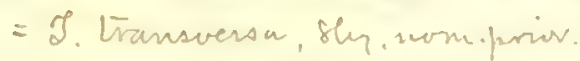

8. add Pholas sagitta, Shears mss.

8<compat>. Photos Pacifier, Learns, al amid lar - flats.

12a Xyiotinga sp. Unalastka, in Mitra spence. 


\section{GEOGRAPHIOAL CATALOGUE.}

1 Lingula albida Hinds.

Santa Barbara, $28 \mathrm{fms}$; San Diego-I. Cal. Calähina y San Miguel 2els- nonterey.

2 Rhynchonella psittacea Limn. ?ns or one of Cidams. Cootes 2 nlet.

3 Terebratula unguiculus $C \rho r .=$ Terebratulina sp. Str. Fuca to San Diego, near 1. w. to 20 fms.

4 Waldheimia pulvinata Gld. P'uret's sil. to Aretic Sea.

\section{4 a Magasella abuctiea, Nall.}

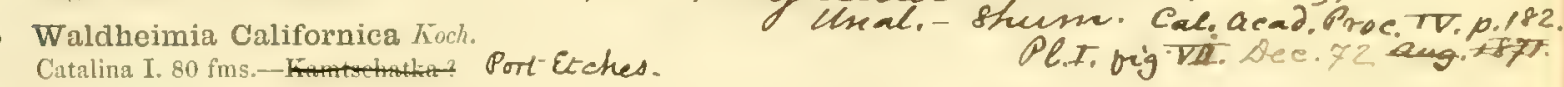

6 Waldheimia Grayi Dav.

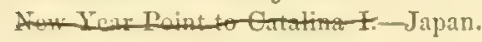

U. 7 Terebratella Coreanica A. Ad. Str. Fuca-China; Japan.

8 Terebratella caurina Gld. = tramsversa, Shy.

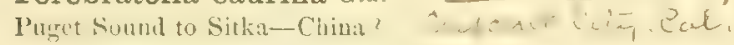

9 Navea Newcombii Tryon. New Y'r Pt. to S. Cruz-L. Cal.? (in IIaliotis.)

10 Xylotrya pennatifera Blainv. Str. Fuca to S. F. Bay-England.

11- Xylotrya fimbriata Jefrey. Str. Fuca-England.

12 Xylotiya setacea Tryon. S. F. Bay (to San Pedro?)

13 Zirphca crispata Linn. Str. Fuca to San Diego--N. Atlantic.

14 Pholadidea penita Conr. Str. Fuca to Santa Barbara, 1, w. cues cent eith, Sheur

15 Pholadidea ovoidea Gld. Baulines Bay to San Diego; rare.

16 Netastomella Darwinii Sby. Str. Fuca to San Diego-S. America.

17 Martesia intercalata $C p r$. Farallone Is. to Mazatlan-(in Maliotis.) 
18 Parapholas Californica Conr. Baulines Bay to San Diego.

19 Saxicava pholadis Limn. Str. Fuca to Santa Barhara-Universal ?

V. 20 Glycimeris generosa Gld. Puget Sound to San Pedro-Kimtschatka.

L. 21 Mya truncata Limn.

Str. Fuca, north-circunpolar-Mass.

22 Platyodon cancellatum Com. Baulines Bay to San Diego.

23 Cryptomya Californica Com. Str. Fuca to San I)iego. Mty + Catahia + Sau niguel 1ds - common -

24 Schizothrerus Nuttalli Con. Str. Fuea to San Diego-Sitka-Japan. Mty.

25 Darina declivis C $p r$. Str. Fuca--California?

26 Corbula luteola $C p r$. San Pedro to San Diego; (color various.) Catalina $\mathcal{L d}$ one valve.

27 Sphœnia ovoidea $C_{p}$. Str. Fuca.

28 Nerera pectinata $C_{p}^{m}$. Str. Fuca to Catalina I. 20-120 fins. Catalina 2d.

29 Clidiophora punctata Cin. Str. Fuca to S. Diegro, 1. W. to 30 fms.

30 Kennerlia filosa $C_{p}$. Str. Fuca. (Aibult of bicarinata?)

31 Kennerlia bicarinata cpr. Catalina I. 40-60 fms. (Young?) Catalina Id.

32 Periploma argentaria Con. Sin P'edro to Sin Diego.

33 Thracia curta Con. Str. Fuca to Sian Dicgo.

34 Cyathodonta undulata Conr. Sta. Barbara to S. Diego-Gulf of Cal. Catalina 2d. unt fide.

35 Iyonsia Californica Conr. Catalina 2), Mumivek

36 Lyonsia nitida Glel. S. F. Bay to San Dicgo. (Young ?)

!. $\quad 37$ Entodesma saxicola lakird. Str. Fuca to Farallone Is.

38 Entodesma infliata Cori. San Pedro, south-l'anama.

39 Mytilimeria Nuttalli Conr. Str. Fuca to San Diego. Whin. 
19. = Siarctica. Arctic deas anio Attu-ian Lrancisco. Catalina $2 d$.

20. Fragments at sitka, 4 fins. ? attu aid Unga -

21. Add var, praecisa, Hld, ano uddevallensis. Sitha, Aratcha. Lall. Plover Bay, Bering fea, geverally. Cake Espent 21a. Mya gapmica Ha... Iapan, Kumchattea, Plover Bay.

25. Ainge plate stanting, no rit, therwise like machäera patula.

27. Ounga 4 frms. Dall. Ounimate Pass. $45 \mathrm{fms}$. Kadiat, Unalashtia miner.

33. Sitka, 1 v. 14 frno, Shumagins to setu.

35. Sitka, 4 fms. $?=+36$. Kyska. ? Ittu.

37. Sitka, abundaut, l.w. Unuluenka.? Kyska. Cat.2d. San niquelorl. 38. inflata Coopor not Connad, = diaphana Per, Magat lan. = spongiophila, Aall, Miss, 1866.

38: Entoderma Seammoni, A all, Fort fimperon, B.C. 



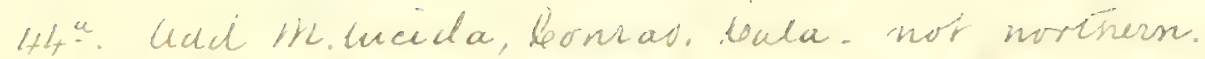

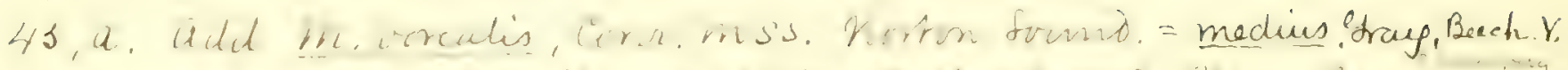

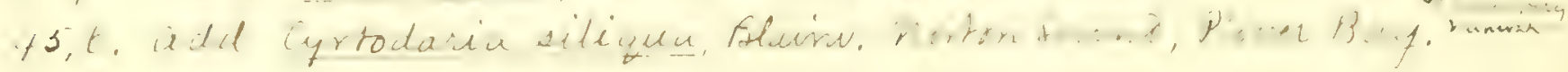

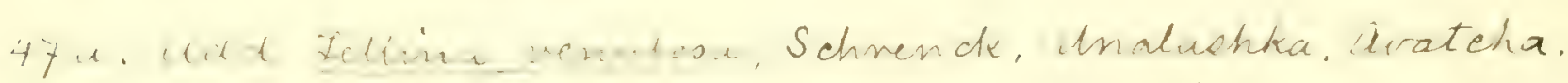

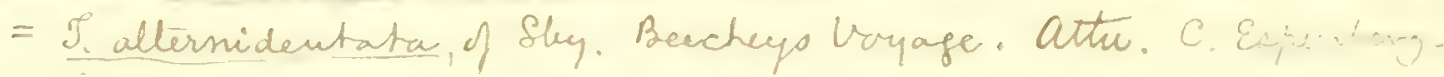
- is huiviak 
40 Plectodon scaber $C p r$.

Catalina I. $10-60$ fms., 2 valves only. Catalina $2 d$ - Living.

41 Solen sicarius Ghd.

Str. Fuca to San Pedro-Japan.

42 Solen rosaceus $C p r$.

Santa Barbara to San Diego. Cal'alina of san Miguel Ids.

43 Solecurtus Californianus Conr. Santa Barbara to San 1)iego. Catalina of $d \delta$ un Migued.

44 Solecurtus subteres Conr. Santa Barbara to San I)iego i=Culifornianus?)

45 Machæra patula Lixon.

Saramna Bay, atu, Kyska te to cala.

46 Sanguinolaria Nuttalli Com. Santa Barbara to San Diego-Japan.

47 Psammobia rubroradiata Com. Str. Fuca to San Diego-Sitka. Petropavtorsk, Kaut. Caltalina $2 d$.

48 Macoma secta Com. Baulines Bay to San Diego-Japan?

49 Macoma edulis Nutt. Str. Fuca to Banl's Bay-Japan? (= secta var ?)

$U: \quad 50$ Macoma indentata $C_{p} r$ Monterey ; (to San Diego ?)

51 Macoma yoldiformis $C_{P} r^{*}$. Str. Fuca to San Pedro.

U.' 52 Macoma nasuta Conr. Str. Fuca to San Diego-Kamtschatka.

cooks inlet.

53 Macoma inquinata Desh. Str. Fuca to Monterey. Estuarics.

7\% $\quad 54$ Macoma edentula Bi:\& Sly.

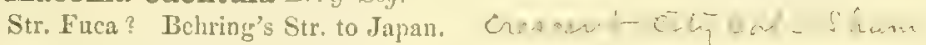

55 Macoma expansa $\mathrm{Cpr}$. Str. Fuca. (=lata Desh. var?)

U 56 Macoma inconspicua $B r . \& . S b y$. Str. Fuca to Santa Barbara-Arctic. C. seant City-Cal.

57 Mera modesta $C p r$. Str. Fuca.

58 Mera obtusa $C_{p r}$. Str. Fuca to San Diego. (=modesta var ?)

59 Mera variegata $C m$. Str. Fuca to Catalina I. Bch-120 fms.

Shumagens to attur Catatina 2d. cooki 2ulet.

60 Mera Gouldii IIant. San Diego-Cerros Is, Lower Cal. Eutacina tet drun thi guei.

61 Mera salmonea $C p r$. Str. Fuca to Montcrey. Bch, to 20 fms. 
62 Tellina Bodegensis IIds. Str. Fuca to San Diecro-Japan.

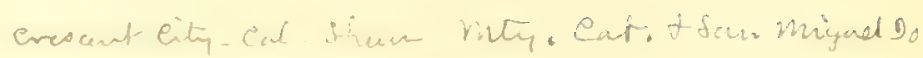

63 Tellinella

San P'dro-2 valves dredgel. (No. 590.)

64 Arcopagia lamellata $C_{\ell} r$. San 1)icgo-Mazatlan.

65 CEdalina subdiaphana (jor. San Diecro ; (Sin l'edro?)

66 Cooperella scintillaformis $C_{l n}$. Santa Burbara to San Diego.

67 Iutricola alta Conr. santa Bauhara to San Diego.

68 Semele decisa Con. Santa Barbara to San 1)iero. Catalina Dd.

69 Semeie xupium sloy. Santa Cruz to lartara Is.-Cialapigos.

To Semele Str. Fuca to Monicrey.

71 Semele rubrolineata Conr. "S. Dicgo, Nuttall." (=decisa var?

7:3 Semele pulchra Sly. Ionterey to S. I) iego-Acapulco to S. America.

i:3 Semele incongrua $\mathrm{Cp}$. Catalina I. 16-40 fms rare.

7.1 Semele flavescens (ild. San Diego?-Mazatlan.

75 Cumingia Californica Comr. Monterey to San I)icgo-Mazatian. Nuty. Catalina, thiquel.

is Donax Califormicus Conr. Monterey? San Imis Obispo to S. Diego.

$\because 7$ Donax flexuosus Gld. Santa liarlara? to San I'edro.

is Donax navicula stoy. S. Pedro? Cape St. Lucas? to S. Americit.

79 Heterodonax bimaculatus $D^{\prime} O, b$. Monterey : S. I'curo to S. Diego-Mex.-W. I.

so Gnathodon mendicum Gild. Culorido estuary to Mazatlan.

S1 Standella Gatifomiea Conr.

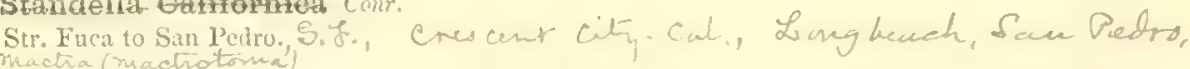

S2 Standella nasuta G/rl.

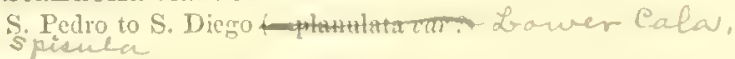

83 Standelle planulata Conr.

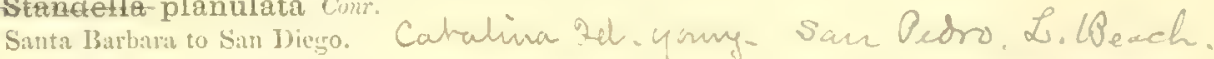
Macha (mactrotoma) californiea Com. Sau Pedro-San sigo. (Lulf?) Stimbla Hamphithi sale. S.Pedro-S. Brèir. 
70. Add S. rubropicta. Dall, Monterey, Santu Erup, foquel 71 . S. sumplex, ad. * Reve, teste Conr. Cat. Catalina d-

$81=+82 ?$ 

90. Sitka, 4 fm.

q2a add Venus

Monterey, sea heach. Neeah Bay, miet, 938

99. A typical Venus, Sitka 14 furs dead. Omit Kamchatka.

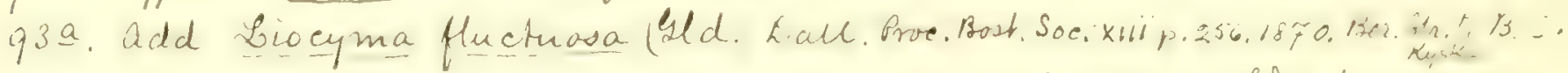

996.

Beckii Dall, l.e. p. 25\%. Plover Bay. Ounga 2D. 4 fm.

$4^{3} \mathrm{c}$

Scanmoni, Dall, Fort fimpson.

$93^{d}$

virichis, Dall, Arctic tecan.

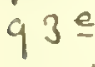

aretica, Rue.

93t. "Mercenaria Kennicotii, tall heah Bay.

95 = Callista sp. 


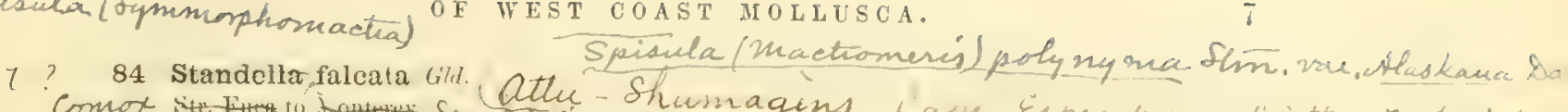

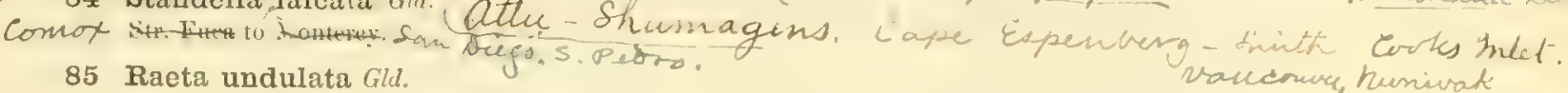

S. Pedro, rare-La Paz, Gulf of California. Cat alina Iel-worth fide, youn-

86 Clementia subdiaphana $C^{\prime} m$.

(Pat elches Btr. Fter-Califormia? Sam Peoro.

87 Amiantis callosa Con.

Santa Barbara to S. Diego-Cape St. Lucas.

88 Pachydesma crassatelloides Conr. Santa Cruz to San Diego. (Str. Fuca?)

89 Psephis tantilla Gld. Str. Fuca to Catalina I., I. W. to 25 fms. Catalina Del- Mhty, San miguel-

90 Psephis Lordi Baird. Str. Fucr to San Diego: (sonthern variety.) Catalina 2d fau higuel.

91 Psephis salmonea $C \rho r$. Catalina I. $30-10$ fms., rare.

92 Psephis tellimyalis $\mathrm{Cpr}$. Farallone Is. or Lower Cal. ? on Haliotis.

93 Mercenaria Kennerleyi Rve. Str. Fuca to Monterey? ? Sutka

4 Meretrix toreuma Gld? Santa Barbara to Catalina I.-- "E. Indies."

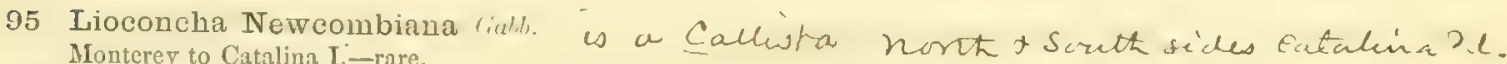
IIonterey to Catalina I--rare.

96 Chione succincta Val. S. Barbara to S. Diego-Mexico-S. America. Catalina $\partial d$ -

97 Chione excavata Cpr. San I'edro to San Diego--(=succincta rar \&)

98 Chione simillima Sby. Monterey to San Diego.

99 Chione fluctifraga Sby. San Pedro to San Diego-Gulf of Cal.

100 Tapes tenerrima $C_{p r}$. Str. Fuca to San Diego-not "Panama." Sauta erup -

101 Tapes laciniata $C m$. Monterey to San Diego-(=staminea var?)

I) $102^{-}$Tapes staminea (uur. Str. Fuca to Sau Diego-l'argarita Bay.

103 Tapes staminea var. Petitii Desh.

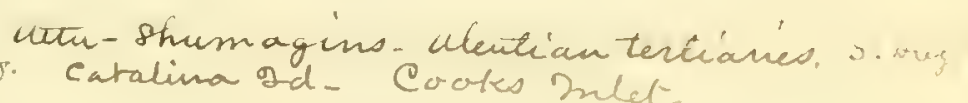
Str. Finca to Monterey-Kamtschatka.

104 Tapes staminea var. ruderata $D_{c s h}$. Str. Fuca to Santa Cruz.

105 Tapes staminea var. diversa Sby. Baulines Bay to San Diego. 
106 Tapes staminea rar. tumida $S b y$.

In cavities of rocks.

107 Tapes staminea 2 rar, orbella $\left(C_{p} r_{0}\right.$ ? $)$

In cavities of rocks.

108 Saxidomus gracilis fild.

Baulines 13ay to Sim 1)ieco- (=aratts (Fllh.?)

109 Saxidomus Nuttalli Com:

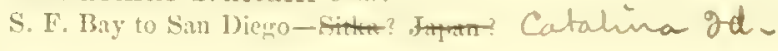

U. $\quad 110$ Saxidomus squalidus $I$ rsh.

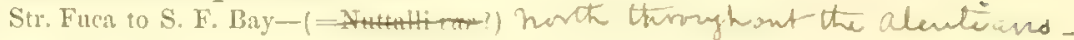

11 Saxidomus brevisiphonatus $C p r$.

Str. Fuen-Vancouver's I.

11: Rupellaria lamellifera Conr.

Farallone Is. to San Diego.

113 Petricola carditoides Cunr.

'Str. Fuca to San Diego-Sitka? Catahima_San Mi guel - Mrä.

114 Chama exogyra Conr.

Baulines Bay to San Diego-Mexico. Catalina-fan míguel.

115 Chama pellucida Shy.

Farallone Is. to San Diego-S. Amcrica. Catalima $2 \lambda$

116 Chama spinosa Sby.

Catalina I. to S. Diego-Mexico, Galaparos.

7) 117 Cardium corbis Martyn.

Str. Fucn to S. Barbarn-Sitka, Kamtschatkn. Catalina 2d? Rumirak

118 Cardium quadragenarium Conr. Monterey to San Diego.

Catalina 20.

110 Cardium blandum cill.

Str. Fuca to Monterey-Sitha; $\Lambda$ sia ?

120 Cardium centifilosum Cjur.

Monterey to Catalina I. 20-40 fims. Catalime 2d-

121 Hemicardium biangulatum Shy. Catahina $2 \rightarrow-C$. ft Lucas -

122 Serripes Groulandicus Chem.

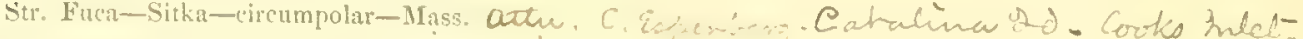

123 Liocardium elatum Sby.

S. Pedro to S. Dietro-Gulf, Mazatlan.

124 Liocardium substriatum Conr. Monterey to Sin I)iego-S. America?

125 Astarte compacta $C_{p r}$. Str. Fuca-(Like compressa of Atlantic.)

126 Astarte Esquimalti Buird. Str. Fuca-Vancourer's I.

127 Astarte fluctuata $C_{p}$.

Catalina I.; (2 valves dredged, 40-60 fms.) 
108. Io this 109 and 110 may probably be added.

112. Kadiak, dead. 7 fur?

114a. Chama inermis, Dall, "Puyet \&d" Rovell, Middle Am.

1.7. ? not corbis Mart from China deas. = Nuttallï Cons. Avatcha, bch.

Crescent city Cal. Schum. Numivate

120. a add Cardium islandicum, Sitka large arid plenty. Madiakte".

121‥ Add Serripes? Daperousei, Srsh. Sitha, Avatcha, Bering Sti. Plover Bay. Iinatashta, attu. StPaul 2d. Nunivak-

124. Astarte sumioulcuta Leach, fite ftü. Dhover Bay. 1246. Astarte mirabitis, Aall, Kadiak. = s.g. Rictocyma. 

132 a Gardita (Ceropsio) minima, Dall $=133$.

133. Irapezium halioticola, Dall, MSS. mty.

137. Sitka, abmoant 7-146mo.

138. Silka, very bine $14 \mathrm{fms}$.

146 a. Leptor rude, Dall, Mty.

1466. Montacutä? Mry. Onll.

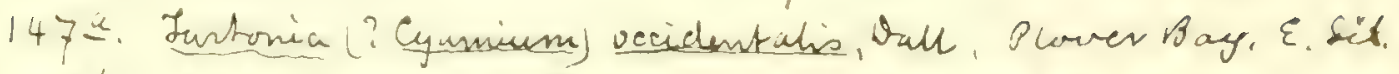

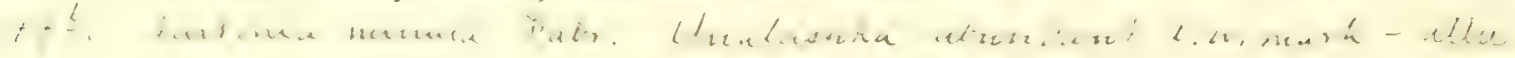




\section{Crassatella}

San Diego, 1 dredged; (No.676.)

129 Miodon prolongatus $C p r$.

Sir. Fuca to Monterey; (dredged.)

V. 130 Venericardia borealis Conr.

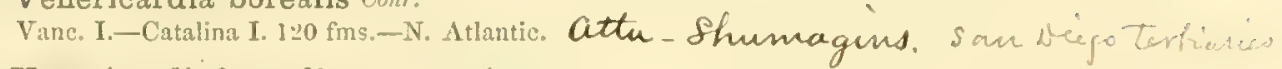

131 Venericardia borealis var. ventricosa

Str. Fuca to Cat. I. 120 f,-S. Barbara. [Gld.

132 Lazaria subquadrata $C p r$. Str. Fuca to San Diega. Catalina - San miguel - Nhy.

133 Trapezium

Monterey ; 1. w. alive in Maliotis-San Diego ?

134 Iucina Nuttalli Conr.

Monterey to San Dicgo. Catalina 2d-

135 Lucina Californica Conr. Santa Cruz to San Diegro.

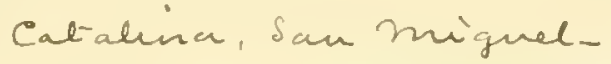

136 Lucina bella Conr.

S. Dicgo, (fossil ?)-Miaatlan.

$Z^{r} \quad 137$ Lucina tenuisculpta $C_{p}$. Str. Fuca to Catalina I,-Mazatlan?

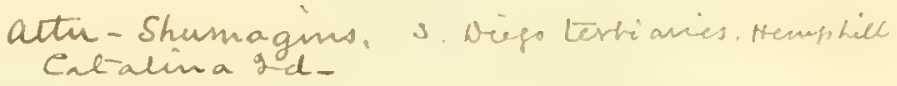

138 Iucina borealis Linn.

Catal. I. 40-120 fms.-N. Atlantic-Asia? Kyska. Sour thiegs Lätiourit

139 Lucina

Monterey.

$\because \quad 140$ Cryptodon flexuosus 3ront.

Catal. I. $120 \mathrm{fms}$ - England-Catalima $2 \mathrm{~d}$.

141 Cryptodon serricatus Cpr.

Str. Fuca-Catal. I. 120 fros. (Distinct sp.?)

142 Diplodonta orbella Gild.

Str. Fuca to S. Diego-in holes of rocks. Cat alina $2 d$ -

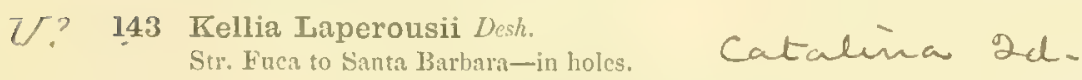

144 Kellia Laperousii var. Chironii $C p r$.

Str. Fuca to San Diego.

145 Kellia rotundata $C p r$.

Monterey; (=Laperousii var?)

146 Kellia suborbicularis Mont.

- Str Fuca to S. Diego-Mex.-Pan,-Europe.

147 Lasea rubra Lont.

Str. Fuca to S. Pedro-All N. hemisphere. attu?

148 Pythina rugifera $C p r$.

Str. Finca.

149 Lepton merœum $C_{p}$ :

MIonterey? S. Diego-shell washings, minute. 
150 Tellimya tumida $(p)$.

Str. Fuca to San Diego.

151 Pristiphora oblonga $C_{j} m$.

San Diego-minute.

152 Sphærium striatinum Lam.

Hell Gate R., Montana? Missouri R. antl cast.

153 Sphærium dentatum Ilahl.

"Oregon;" Nuttall coll., 1831.

154 Sphærium occidentale Prime.

Hell Gate K., Montann-Listem states.

155 Sphrrium nobile Goukl.

Near San Pedro, Cal., or Colorado River.

156 Sphærium patella Gild.

"Oregon;" U. S. Expl. Iixpl'n coll., 1847.

157 Sphrerium lenticula Gild.

Lake 'Ialıo and Carson River: Krlamath River.

158 Sphrerium tumidum Buird. Sumass R., lat. 49 , IV. T'.

159 Sphærium Spokani Bairl. Spokan R. to Kootenai R. lat. 49 , W. T.

160 Pisidium compressum P'rime. "California"-Nurth $\Delta$ tlantic States.

161 Pisidium abditum Huld. "California"- Atlantic states.

162 Pisidium occidentale Vewr. Merced Lake, S. F. to Santa Cruz, Cal.

163 Pisidium ultramontanum l'rime. Canoe Creck, Pit River, Califumia.

164 Unio Oregonensis Ler. "Columbia Iiver," Wheatley Mluscum.

165 Margaritana falcata Gill. s. Cruz, California, north-Nevada; Montana.

166 Anodonta angulata Lut. San Joaguin R., north; Idaho; Montana.

167 Anodonta Californiensis Left. Inland streams of Cal.-Colorado River.

168 Anodonta Nuttalliana Loa. Willamette liver; Ilaho-Vancouver's I. ?

169 Anodonta Oregonensis Lor. Near San Franciaco; Oregon; Montana.

170 Anodonta Wahlamatensis Lea. Uner Sacramento R.; Idaho to British Cul

171 Anodonta Kennerleyi I r s. Lake Chilowernet, lat. 49 , W. 'T. 
163 a add Pisidiurn Nasfortionnm, Prime, MSS. 


180․ Modiola aterrima, Dall, gipan.

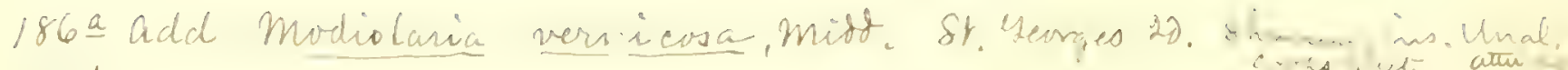
$1866^{\circ}$." denticulata. Dall, Acapulco.

192 a Nucula exigua dowerby, drift, dan Peclro, Shepard.

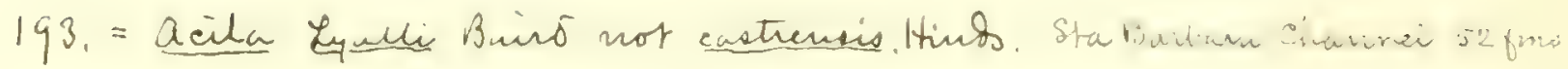


172 Mytilus Californianus Conr.

Str. Fuca to San Diego-Q. Charlotte's I. Shumagins. Crescent city. Cu

Catalina - S. Miguel

173 Mytilus edulis Linn.

Monterey, north-Japan; N. Atlantic. Universal C.Es pe:ch-

17. Mytilus edulis var. glomeratus Gld.

S. F. Bay, on alga-deformerl?

175 Septifer bifurcatus live. [Conr.]

Farallone Is. to San Diego. (Not Myt.bifurc. Catalina 2l. Cal.

176 Modiola capax Conr.

S. Barbara to S. Diego-Lower Cal. and Mex.

$l^{-}$

177 Modiola modiolus Lin.

Monterey, north-Asia; N. Atlantic.

uniressal

178 Modiola fornicata $C p r$. Monterey to San Pedro.

179 Modiola recta Conr. Santa Cruz to San Diego.

\section{S. Dijo terticine}

180 Modiola recta var. Hlabellata Gld. Str. of Fuca.

181 Adula falcata Gld.

San Francisco to San Diego-New Zealand?

182 Adula styliua $C p r$. Str. Fuca to Monterey.

183 Lithophagus plumula Hanl. Monterey to S. Diego-Mexico to Panama.

184 Lithophagus attenuatus Desh. Monterey, south? -Lower Cal. to S. America.

7.- 185 Modiolaria Iævigata Gray. Str. Fuca-Japan; N. Atlantic. Wtu-Shumagins. Numivak

186 Modiolaria marmorata Fls. Str. Fuca-Europe.

137 Crenella decussata Mont. Catalina I. 10-40 fms.-North Europe.

Kyska

188 Barbatia gradata, Sby.

S. Barbara to S. Diego-Japan; Mex. and S. A.

189 Axinga intermedia $B$ rod.? Monterey to San Dicgo-South America. Cataliua 2d.

190 Axinæa septentrionalis Midd.

- Sitka to Aliaska; "Ukamok I." lat. 64.

191 Axinza subobsoleta $C p r$. Str. Fuca to Farallone Is. (=septent. var?)

C. 192 Nucula tenuis Mont. Str. Fuca to S. Barbara-Japan; N. Atlantic.

193 Acila castrensis Minds. Str. Fuca to San Diego-Sitka. 
194 Leda crelata Hinds.

Bodega Bay 10 San Diego; 6-20 fins. Cataliuna sj)

195 Leda cuneata Sby.?

Monterey to San Diego-Peru.

190 Leda minuta O. Falr. Str. Fuca-N. Atlantic.

' 197 Leda fossa Luird. Str. Fuca-Vanc. I. 10-15 finc.

198 Leda hamata $C_{y} m$. Santa Larbara I6--28 fms. Catalinis I i J

?. 199 Yoldia lanceclata .J. Sty Str. Fuca-Japran; N. Atlantic :

200 Yoldia amygdala Str. Fuca-Monterey 40 fins.

201 Yoldia Cooperi Gabl. Santa Cruz to San Pedro--Bearch, vallyes.

202 Verticordia ornata LO Orl. $^{2}$ Santa Barbara, 28 fnss.-Japan?; Peru? Cat alina ded.

203 Philobrya setosa $C_{p}$. Santar Barbara-Capre St. Lucas; Acapuleo, Catalima : $)$.

204 Lima dehiscens Conr. Monterey to S.Diego-(=orientalis $\mathbf{A}$ ? China.) Catahina. Sam miguel.

205 Limatula subauriculata .hont. Catalina I. to San Diecro-N. Atlantic. Catalima 2d.

206 Pecten hastatus Sl,y. Str. Fuca to Sinta Barbara-nitka; Aliaska. Cooks 2nlet St Daul $2 d$.

207 Pecten hastatus ver. Hindsii $C_{p}$. Str. Fuca to sintil Cruz--Sitkin.

208 Pecten paucicostatus $C_{p}^{\prime \prime}$ Senta Barbara (to San Pelro?) rar? Cataliono $2 d$ -

209 Pecten aquisulcatus Cip: Montcrey to San Diego (=ventricosus zur?)

210 Pecten latiauritus Com. Monterey to San Dicon.

211 Pecten monotimeris ('onr. Monterey to San Diego; (=]atiauritus rat?) Catalina 2d.

212. Amusium caurinum G/l. Str. Fuca (to Santa Barbara?)--Jin)an.

213 Janira dentata Sty. Monterey to Sin Diero-Gult California. Cabaluma $2 d$

214 Hinnites gigantens (roy.

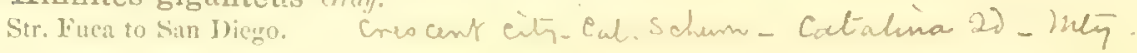

215 Ostrea lurida C $m$. sitr. Fuca to sim 1)ieno. 
199. 2oanotaky Pass, Kadiak, Unga.

200ミ. Foldia seminuda, Aale, st Pauls Hartor, Kadiak, I:

2006 . Yoldia Thaciaejormis, Stin, Unalashke.

200 Goldia monhereyendis dael, monterey, Kifi.e.

211 - Pecten Alaskensis. Dall, Sitka, Unga, Kadiak, Port Eides.

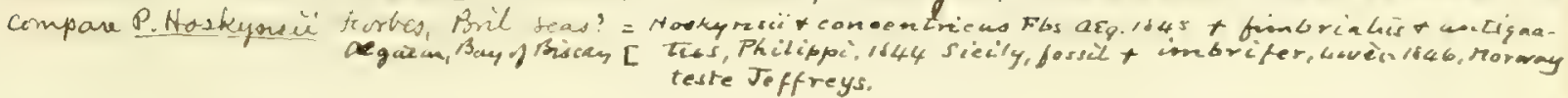

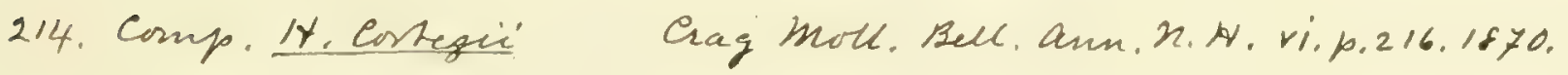




222.? not of Limme.

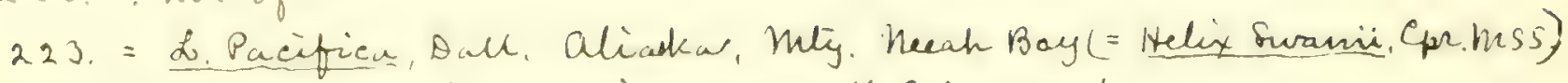
223 - Clevora sccidentalis. Aall off Culn. Corast.

223․ Corolla spectabilis, Dall, near Alentian 2slands.

2235 Clione eleguntiosina, Dall.

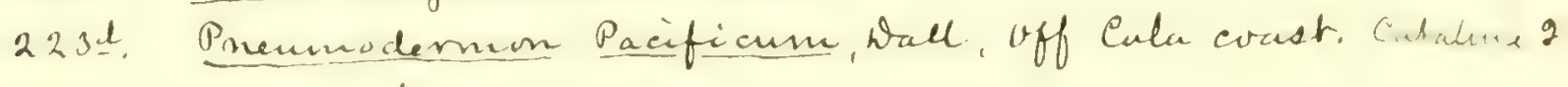
Also atlanta turo op.

Carimaria

Salpa herenlea, Wall, Mnimak Pass.

" eymbive, Dall.

Boltenia Beningi, Dall. St. Hearges 2d. Nunivak. Cynthia pyriformis, Lim. Plover Bay, Autcha. monterayensis, Dall, mity.

Sprenoicularia

tied mannia

If Pr Conception.

235a. add Jornatina harpa Dall. mss. Mly. Vichoria, newembe. 
216 Ostrea (lurida var?) laticaudata $C_{p}$ San Francisco Bay to San Diego.

217 Ostrea conchaphila Cur. Catalina I. to Panama.

218 Ostrea (conchaph. var?) expansa Cpr. San Diego-Margarita Bay.

219 Ostrea (conchaph, var?) rufoides $C_{p r}$. San Diego.

7. 220 Placunanomia macroschisma Lesh. SL:Paed od. Str. Fuca to San Diego-Japan.

221 Anomia lampe Gray.

"Monterey, 60 fms." San Pedro to Mexico.

222 Cavolina telemus Lim.

Sir. Fuca to Catalina I. Pelagic.

223 Limacina arctica Fabr.

Monterey?-N. Pacitic; N. Atlantic.

224 Bulla nebulosa Gld.

Santa Barbara to S. Diego-Xower Cal-Gulf. Catalina $2 d$ -

225 Bulla Quoyi Gray.

S. Barbara to S. Diego ?-Mex. "N. Zealund."

226 Bulla Adamsii Mhe.

San P'edro? to Panama.

227 Haminea hydatis Lim.

Str. Fuca--Europe.

228 Haminea vesicula Ghl.

Santa Barbara to San Diego--Cape St, Lucas.

229 Haminea virescens Sby. Catalina I. to San Diego.

230 Atys nonscripta 4 . Ad. San Diegro; (original locality unknown.)

231 Tornatella punctocelata $C_{p}$. Santa Cruz to San Diego. rium

$\ell^{-}$. 232 Tornatina culcitella Gll. Monterey to San Diego. Catalina 20. Sta Barbera chan -

233 Tornatina cerealis Gld.

Munterey to San Diego.

234 Tornatina eximia Buirl. Monterey?-Vanc. I. $12 \mathrm{fms}$.

235 Tornatina carinata $C p r$. Santa Barbara to San Diego; Panama.

236 Cylichna cylindracea Linn. Catalina 2d. Monterey to San Diegro-Europe.

237 Cylichna (cyl. var?) attonsa $C_{p}$. Sir. Fuca to Sinta Barluara.

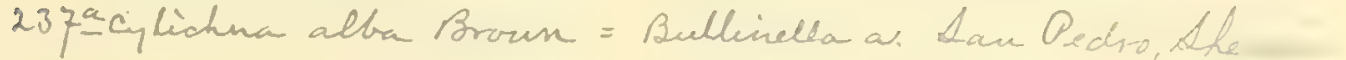


238 Cylichna planata Cyr. San 1)iego. (Cassilly.)

239 Cylichna inculta Gikl. Sian Diegro. (Blake?)

240 Volvula cylindrica c pn. Sinta Barliara. (Jewett.)

241 Neaplysia Californica $C_{l}$ Munterey to San Pedro.

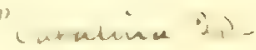

242 Navarchus inermis $C_{p}$. Catalina I. to San Diego.

243 Pleurophyllidea Californica $C_{P}$. San I)icro-Vancourer's I.

244 Doris sanguinea $(\%$. Barlara I. to S. Diegu.

245 Doris alabastina $C_{p}$. San Dicgo; 1 specimen, 1. w.

246 Doris albopunctata $C_{p}$. Baulines Bay to Catalina I.

247 Doris Sandiegensis $C_{p}$. Baulines Bay to San Diegro.

248 Doris Montereyensis $C_{p}$. San Francisco Bay to Barbara I.

249 Triopa Catalinx $C p$. Baulines Bay to Catalina I.

250 Tritonia Palmeri $C p$. San 1)iegro, 1. w., sand, in December.

251 Dendronotus iris $C_{p}$. Santa burbara clannel; in kelp.

252 ZColis Barbarensis $C_{p}$. Santa laurbara licach, on algar.

253 Phidiana iodinea $C p$. Santa Cruz to San Diego.

254 Flabellina opalescens $C_{p}$. Monterey to San J)iego.

255 Chioræra leonina Gith Str. Fuca-Santa Barbara?

256 Ariolimax Columbianus Ghl. Str. Iuca to Santa Barbara.

257 Arion foliolatus Gikl. P'uget's sound conntry--California ?

258 Succinea Stretchiana Blame. Near Lake Tabo, Sierra Nevadia.

259 Succinea Gabbii Trym. Northenst California and Southeast Oregon. 
240. Montery.

2439. Laterivanchata fectiva, Stearns, Mty. Catulina 2d.

249a. Hiopa Carpenteri, Shearns, Mety.

255․ albopapillwow, Dall Sicka.

255\%. Onchidella borealis, Sall, Sitka. Port Etches. 

475 . Niputina chersinglla. 
260 Succinea Hawkinsii Baird.

Near lat. 49, Wash. 'Territory.

261 Succinea rusticana Gll.

Oregon to Tulare Valley, Cal.

262 Succinea Nuttalliana lea.

Wright's Lake, Cal, to Snake R. Oregon.

263 Succinea lineata W. G. Bin.

Northeast Cal, to Nebraska and Brit. Col.

264 Succinea Oregonensis $L<a$.

Oregon to Santa Cruz, Cal.

265 Succinea Sillimani Bland.

Ifumboldt Lake, Nevada.

266 Vitrina Pfeifferi Neuc.

Carson Valley, Nev, to Owen's Vailcy, Cal.

267 Binneya notabilis $C p$.

IBarbara I. Cal. ; southeast side onfy.

268 Hyalina arborea Say.

San Gorg. Pass, Cal.-Montana--E. States.

269 Hyalina Breweri Neu'c.

"Big Trees," Calaveras Co., north.

270 Macrocyclis Newberryana I'.G.Binn.

Terescal Mlountains, near San Diego.

271 Macrocyclis Vancouverensis Lea.

Santa Cruz, Cal, north; Idaho.

272 Macrocyclis sportella Gld.

Shasta Co., Cal,, to P'uget's Sound.

273 Macrocycils Voyana Newc.

Cañon Creek, Trinity Co., Cal.

274 Conulus chersina Say.

San Gorgonio Pass to Iake Taho-E. States. Athe, Petropuirt ... .

275 Conulus? chersinella Dall.

"Big Trees," Calaveras Co., Cal.

276 Pseudohyalina milium Morse?

Monterey to Nerada Co., Cal.--Maine.

277 Pseudohyalina minuscula Binn.

San Bernardino, eastwarl to Maine-IV. I.

278 Pseudohyalina conspecta Bland. San Francisco; Monterey.

279 Patuila Hornii Cácbl. Fort Grant, Arizona.

280 Patula Whitneyi Newc. Near Lake Taho, California.

281 Patula Cronkhitei Neur.

Alameda Co., Cal., to Kilamath Lake. 
35. Patula striatella A $u$ h.

Montana?-Worthern States to Slave Iake.

¿8:3 Patula Durantii Yeure.

Barbara I., Cal. ; southeast side ouly.

uSi Helicodiseus? polygyrella B/d. Bitter-root Mountains, Montana; in moss.

255 Anguispira solitaria Say. Montana? Missouri to Lake Erie.

286 Anguispira Cooperi $W^{r}$. G. Bim. Montana to Arizona; in mountains.

257 Anguispira strigosa (ild. Nonfan to Arizona; in mountains.

238 Anguispira? Idahoensis Nerc. Fastern Itaho; in monntains.

339 Helix Hillebrandi Nenc. Mariposn and 'luolumne Cos., Cal.

290 Helix Mormonum Pfeif Fresno Co. to Shasta Cu., Carl.

291 Helix sequoicola $C_{p}$. Santa Cruz Co., Cal.

292 Helix infumata Gild. Contra Costa Co. to Mendocino Co. Cial.

293 Helix fidelis Gray. II umboldt Bay, Cal., to Vancourer's I.

294 Helix Dupetithouarsi Desh. Monterey, (al. (Eoint Cypress.)

295 Helix Áviesiana Nekc. Sinta Cruz I., Cal. (Newcomb Coll.)

200 Helix Traskii Nexr. Near Los Angeles to San Luis Obispo, Cal.

297 Helix rufocincta Nerc. Catalina I., Cal.; Sayt Dint?

298 Helix Gabbii Neur. Clemente I., Cal.; Gntaltint I?

299 Helix facta Nerce. Barbara and Nicolis Is. Cal.

300 Helix Rowellii Nur?. "Arizona?" (Frick.)

301 Helix Carpenteri Newc. Tulare Valley. Very rure.

302 Helix Tryoni Nerc. Barbara and Nicolus Is., Cal.

303 Helix intercisa I'. G. Bim. "Oregon"--San Mlignel I., Cal. ? 
291 . hylvid of Bupetithouarsi?

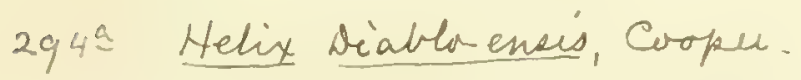




319. 2s certainly the tame as 318. 
304 Helix crebristriata Nerc.

Clemente I.; San Miguel I., Cal. ?

305 Helix Kellettii F'bs.

Catalina I., San Diego and soutl.

306 Helix Californiensis Lere. Monterey, Cal. Rare.

307 Helix redimita W. (\%. Binn. San Francisco to Monterey.

308 Helix Nickliniana, Ler. Santa Cruz to Mendocino Co., Cal.

309 Helix Bridgesii Nerc.

Contra Costa Co., Cal. (=Nickl. wer.?

310 Helix arrosa Gild.

Santa Cruz to Mendocino Cu., Cal.

311 Helix exarata Pfeiff.

Santa Cruz Co., Cal. ; Marin Co. ?

312 Helix ramentosa Gld.

Napa Co. to Santa Clara Co., Cal.

313 Helix reticulata Pfeif:

Locality unknown; (=ramentosa var?)

314 Helix tudiculata Bim.

Placer Co. to S. Diego, Cal.; Oregon? Sunta Bistura-Baken.smoot var.

315 Helix Townsendiana $L e \alpha$.

Crescent City, Cal., north; Montana.

316 Helix anachoreta $W . G$. Bimn.

California? Oregon. (Rowell Mus.)

317 Odotropis devia Gld.

Oregon to Vancouver's I.

318 Aplodon Columbiana Lea.

Wash. Territory to Santa Cruz, Call. Sitka St. Seerge A Paul Ods?

. 319 Aplodon germana Gld.

"Oregon." Probably =Columb. var.

320 Triodopsis Mullani Bld.

Western Mountains of Montana.

321 Triodopsis loricata Gld.

Near San Francisco to Filamath Cu., Cal.

322 Pupa hordacea Gabb.

Fort Grant, Arizons.

323 Pupa Arizonensis Gabb.

Fort Grant, Arizona.

324 Isthmia corpulenta Morse.

Lake 'Taho to Washoe Co., Nev.

325 Pupilla Californica Rowell

Near San Fraacisco to Montercy. 
:36 Pupilla Rowellii Newe Near Uakland, Alameda Co.

327 Pedipes unisulcata $C p$. Sin Pedro, Call; on licacl.

328 Pedipes lirata $W^{*}$. ( $x$, Bim. Cane St. Lueas, Lower Cal._Sau biego?

329 Melampus olivaceus (yr. Salinas River, Cal., to Mazatian.

330 Limnaea stagnalis Limn. liuby Valley, Utah, north--circumboreat.

331 Limnæa lepida Gld. "Lake Vancourer," Wash. 'I'er. ; Uremon.

332 Limnophysa Rowellii Tryon. Near Sam Franciseo.

333 Limnophysa palustris .Mull. Near sin Francisen-cireumboreal.

334 Iimnophysa proxima Lru. Alameda County, Cal., to Oregon.

335 Limnophysa Gabbii Trym. Clear Lake, California.

336 Limnophysa Adelinæ Tryon. Near San Fraucisco, California.

337 Limnophysa Traskii Tryon. Mountuin Lake, San Francisco Co, Cal.

338 Limnophysa umbrosa Say. Near San Francisco, Cal, ; Eastem States.

339 Limnophysa bulimoides Lea. San Francisco, north and east; S. Diego?

\$40 Limnophysa solida $L \ll$. Willanette River, Oregon.

341 Limnophysa ferruginea IIald. "Oregon." (=lumilis Say. ?)

342 Limnophysa humilis Sing. Nlameda County, Cal.; Lustern States.

343 Limnophysa Sumassi Buird. Oregon to British Columbia.

344 Limnophysa Biuneyi Tym. "Hell Gate liver," Montana.

345 Limnophysa Nuttalliana $L(c)$ San Francisco to Oregon.

346 Limnophysa Tryonii Leu. Alameda County, California.

347 Limnophysa obrussa Sim. "Oaklanil," ('al.; Easıern States. 





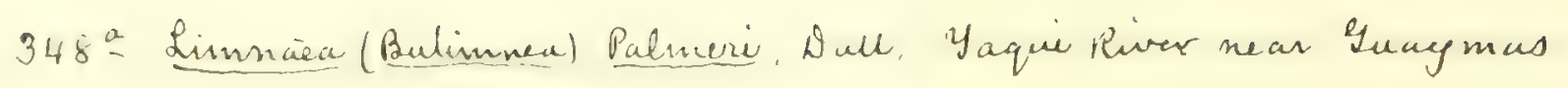

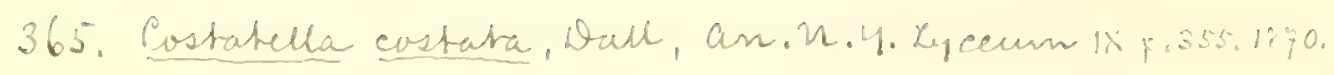


348 Limnophysa caperata Say. "S. F. to Oregon." Eastern States.

349 Acella

Spring on Spokan Plain, W. T.

350 Physa Grosvenorii Lea.

Dayton, Nevada. (Wheatley Mus.)-Kansas.

351 Physa coniformis Tryon.

Humboldt River, Nevada.

352 Physa Cooperii Tryon.

Crane Lake Valley, northeast Cal.

353 Physa sparsestriata Tryon. S.n Joaquin Valley, Cal.

354 Physa diaphana Tryon. Oakland, Alameda Co., Cal.

355 Physa malleata Tryon. Goose Lake Valley, N. E. Cal.-Montana.

356 Physa Nuttallii Lea.

Lewis River, Idaho.

357 Physa distinguenda Tryon. Marysville to Stockton, Cal.

358 Physa politissima Tryon. Sacramento, California.

359 Physa parva Lea. Little Valley, Nev. (Wheatley Mus.)

360 Physa propinqua Tryon. Jordan Creek, southwest Idaho.

361 Physa gyrina Say. Carson River, Nev.; Utah. Lustern States.

362 Physa ampullacea Gld. Lake Osoyoos, lat. 49, Wasl. Ter.

363 Physa occidentalis Tryon. Most of Cal. northward; Idaho.

361 Physa Gabbii Tryon. Mountain Like, S. F. Co., to Los Angeles.

365 Physa costata Newc. Clear Lake, California.

366 Physa virginea Gld.

- Sicramento River, Cal.; Sim Francisco?

367 Physa venusta Lea.

Near Vancouver, Columbia R., IV. T.

368 Physa humerosa Gld. Colorado R., Cal.; Pecos R., Texas.

369 Physa virgata Gild. S. Pedro to S. I)iego; Mojave R. to Gila R. 
370 Physa triticea $L c a$.

Shasta Co., Cal.; South and East Oregon.

371 Physa concolor Liald.

"Oregon"-probably young of another.

372 Physa Traskii Lea.

Los Angeles River, Cal.

373 Physa Blandii Lea.

Marysville, California.

374 Aplexus hypnorum Lim.

Montana? Russian Am.--circumborenl.

375 Aplexus hordaceus Lea.

Near I'uget's Sound, W. 'T.; Vancouver's I.

376 Planorbis subcrenatus $C p r$.

Oregon: Wash. Ter.? Vancouver's I.

377 Planorbis gracilentus Gld.

Colorado Desert, California.

378 Planorbis tumens $C_{\mu r}$.

San Francisco to I'etaluma; Mazatlan.

379 Planorbis glabratus Say.

New Mexico? (=sinuosus Bourg.?)

380 Planorbis Oregonensis Tryon.

Nevada; Oregon; Idaho. (Hot spring.)

381 Gyraulus vermicularis Glel.

Walla Walla, W. T.; Uremon.

382 Gyraulus parvus Stry.

Ruby Valley, Utah; Montana; Linstern States.

383 Helisoma ammon Gld.

Colorado IR.; Kern Lake to Kklamath Lake.

384 Helisoma corpulentus Suy.

Columbia River, cast to Lake Winipeg.

385 Helisoma trivolvis Say.

Whole United States; Cuba.

386 Menetus opercularis Gld.

Santa Cruz, Cal., to Str. Fuce, W. 'T.

387 Carinifex Newberryi $L_{\ell} \alpha$.

Clear Lake, Call, to Klamath Lahe, Ol.

388 Pompholyx effusa $L \mathrm{cr}$.

Carson River, Nev, to Pit River, Cal.

389 Ancylus Kootaniensis Bairr. Spokan to Kootanie River, W. T.

390 Ancylus altus Tryon.

Klamath Iiver, California.

391 Ancylus Newberryi $L_{c a}$.

Iklamath Lake, Oregon. 




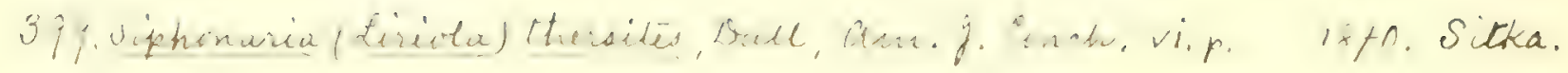
3999. Ald Siph. Liriola) peltoides, Cpr. Mty. Mag. Catalina, Salapagus. This may be an anisomyon, ans is Canfieldia, epr.mss.

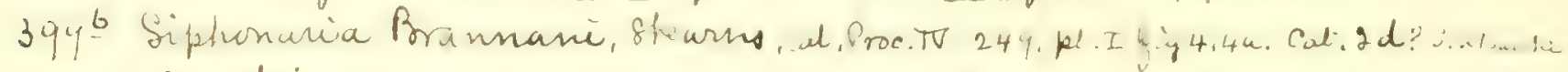
400 a Tanthina a. Ad. see H.Knocker, R. W paper on Otewo pods. D.2.S. $402 .=401 ?$

408. Ionicella lineatä, Cpr. 
392 Ancylus crassus II ald.

"Oregon." (Nuttall coll., 1834.)

393 Ancylus caurinus $W . C p$. Black River, I'uget's Sound, WV. T.

394. Ancylus patelloides Lca. San Francisco to Santa Cruz, Cal.

395 Ancylus fragilis Tryon. Laguna Ilonda, near San Francisco.

396 Ancylus subrotundatus Tryon. Umpqua River, Oregon.

397 Ancylus Nuttallii Hald. "Oregon." (Niuttall coll. 1834.)

398 Gundlachia Californica Rowl Feather River, Cal., near mouth.

399 Siphonaria thersites $C \mathrm{~m}$. Neeah Bay; Str. Fuca, IV. 'T.

400 Ianthina globosa Sw.?

S. Diego to Cape St. Lucas-Atlantic.

401 Dentalium pretiosum $N$ utt. "California," (Nuttall.) Mexico?

402 Dentalium Indianorum $C p r$. Str. Fuca to Santa Barbara. Monterey-

403 Dentalium rectius $\mathrm{Cpr}$. Str. Fuca.

404 Dentalium semipolitum $B r$. and Sly. San Diego to Gulf of California.

405 Dentalium hexagonum Sby. Santa Barbara to Mexico-_" E. Indics, China."

406 Crypochiton Stelleri Midd.

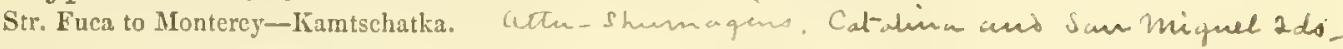

407 Katherina tunicata Wood. Str. Fuca to Monterey-Sitka. Cooks inlet - Unalashka - altu-

(f. 408 Tonicia lineata Wood. Str. Fuca to Monterey-Sitka; Kímtschatka.

409 Tonicia submarmorea Midd. Str. Fuca to Oregon-Kamtschatka.

410 Mopalia muscosa Gld. .Str. Fuca to San Diego.

411 Mopalia Wossnessenskii Midd. Str. Fuca to Santa Cruz-Sitka.

412 Mopalia Kennerleyi $C p r$. Str. Fuca, W. T.

U. 413 Mopalia Kenuerleyivar? Swannii $C p$. Neeah Bay, W. T. 
414 Mopalia Hindsii Gray. Str. Fituca to Monterey.

415 Mopalia Simpsonii Gray. San Francisco, California.

416 Mopalia vespertina Gle. Str. Fuca to Monterey, Cal.

417 Mopalia lignosa Gld. Str. Fuca to Monterey-Sitka.

418 Mopalia acuta Cpr. "Montcrey," (Nuttall coll.)

419 Mopalia? sinuata $C p r$. Str. Fuea, W. T.

420 Mopalia? imporcata Cpr. Sir. Fued, W. T.

421 Acanthopleura scabra live. Str. Fuca to Santa Barbara.

422 Acanthopleura fluxa Cpr. Catalina I., or Sinta Barbara I., Cal.

423 Ischnochiton Magdalensis Hus. Montercy to San Diego-Lower Cal.

424 Ischnochiton veredentiens $C_{p r}$. Monterey, or islands oft South California.

425 Lepidopleurus regularis (pr. Monterey or southward.

426 Lepidopleurus scabricostatus $C_{j}$ r. Catalina I., 20 fins., rare.

427 Lepidopleurus pectinatus $C_{p}$. Catalina I., 1. w., rare.

428 Lepidopleurus Mertensii Midd. Str. Fuca to Monterey.

429 Trachydermon retiporosus C'pr. Ser. Fuca, IV. 'I'.

30 Trachydermon interstinctus cill. l'uget's Sound to Monterey.

431 Trachydermon trifidus $C p r$. Str. Fuca, W. T.

432 Trachydermon dentiens Gld. Puget's Sound, W. T.'?

433 Trachydermon pseudodentiens Cpr. Str. Fuca, IV, 'T',

434 Trachydermon gothicus Cpr. Catalina I., 20 fms., l. w.?

435 Trachydermon Hartwegii $C_{p}{ }^{\circ}$. San Francisco to Monterey. 
417. 2o M.merckii, midd.

421. 2s Nuttallina scalra, epr.

423. 20 Lepidoradsia magdalenensis, Cpr.

425. Is 2schnoradsia regularis, epr. add Ischnoradria trifida, Epr. Sitka.

4349 add Irachydermonfallax Epe.

435. Is Châtopleura Harturgici, Cpr.

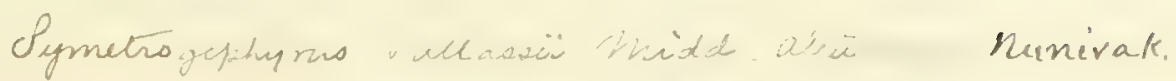
add Callistochiton fimbriatus, Cer.

Leptochiton negatus, Cer.

Placiphorella lation, Cpr.

Chactoplenra genma, epe.

2echnochition rabians, epe.

2schnochiton Covperi, Cper. 



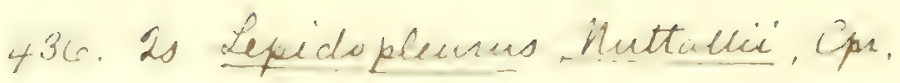

439. 20 Leptochiton internexus, Cir.

441. 20 not a Nacella. Crescent Giti. Sal. Sehuse

442. 2s a tupical Acmaea.

443. 20 an abnormal Lirida peltoides Cpr. from examination of the type.

444. This and the two following do not loleng to the gens Nacella but are probably Acmaeids.

445 2s a collisella.

446. Cirnp. Patellu pyzmisea, trumk. 'm Ml, fup.p. p.24 pl. III fig. 20. The normal var. is casta Cpr. mss. probably a Collisella.

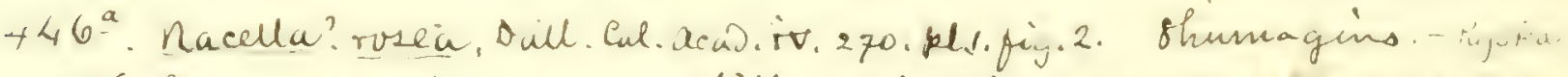

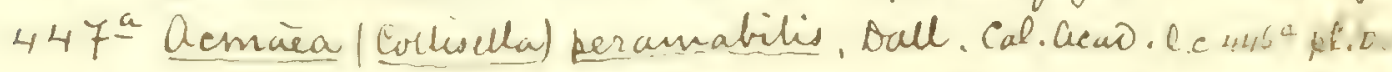

448. Acmiace, subyerus collisellu, wall. Add var ochraces, fall.

449. Jppe of s.ba. Erliserea. Add var nacelloides, Dall. in.i.t.

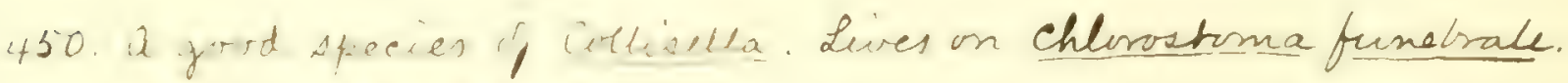
45\%. Collisella pereona.

452. Collisulla scabra. Sitka? V. Id. Kennientt.

453. Collisella spectrum.

454. This species? was protably foundid on a young pelta. What it might have been is now inpossible to determine. 455. a govt species.

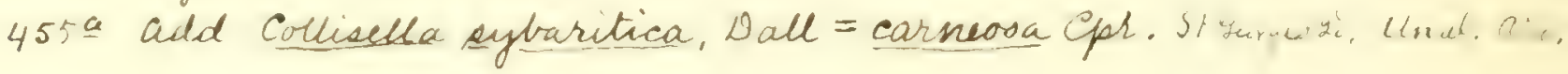

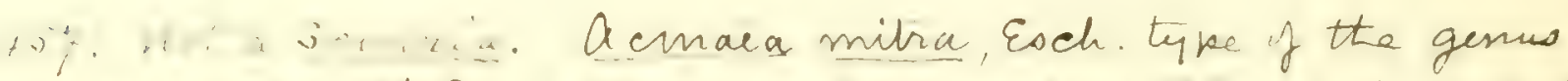

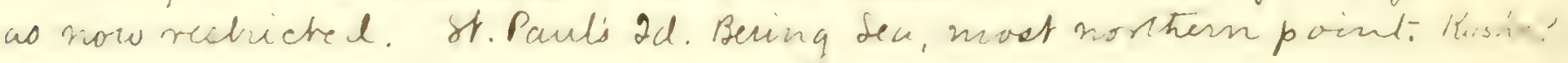


436 Trachydermon Nuttallii Cpr. ? = ime of $433+435-$ Cpar. Str. Fuca to Monterey.

437 Trachydermon flectens $C p r$.

Str. Fuca to San Diego.

438 Callochiton fimbriatus $\mathrm{Cpr}$, (Mss.)

Catalina (or other?) I., Cal.

439 Leptochiton nexus $C p r$. Catalina Is, Cal.

440 Acanthochites avicula $C p r$. Monterey? Catalina I. 8-20 fms.

441 Nacella instabilis Gild. Str. Fuca to Monterey.

442 Nacella insessa $H d s$. Baulines Bay to San Diego. Catalina dd. Mty. San Mniguel.

443 Nooella subspiratis Gir. Catalina I. 6-10 fms.

444 Nacella depicta IIds. Santa Barbara to San Diego.

445 Nacella paleacea Gld. Monterey to Santa Barbara.

446 Nacella triangularis $C p r$. Baulines Bay to Monterey.

447 Tylodina fungina Gabb. Barbara I., Cal. Sau Pedro Shepan, San Diego Mise Luve.

U. 448 Acmæa patina Esch. Str. Fuca to San Diego-Mazatlan-Sitkar-atu. Cresent eily- Kemai pun.

U. 449 Acmæa pelta $E$ sch. Str. Fuca to San Diego-Sitka.- attru. Kenaifu. Cresent city.

450 Acmæa pelta var. Asmi Midd. Baulines Bay to Monterey-Sitka! Catahna 2d.

U 451 Acmæa persona Esch. Str. Fuca to San Diego-Mazatlan-Sitka. Kenai

452 Acmæa scabra Nutt. Baulines Bay to San Diego--Mazatlan.

453 Acmæa spectrum Nutt. Cape Mendocino to San Diego.

454 Acmæa pileolus Midd. Sitka.

455 Acmæa pileot rosacea $\mathrm{Cm}$. Monterey to Santa Barbara. Catalina 20.

456 Lottia gigantea Gray. (Sby. ?) Farallone Is. to San Diego-S. A.? Catalina Dd -

1\%. 457 Scurria mitra Esch. Str. Fuca to San Diego-Sitka.

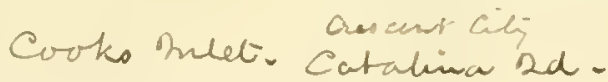


458 Scurria mitra var? funiculata $C p r$.

Monterey; deep water.

$7^{-} 459$ Lepeta cœcoides $C_{p r}$.

Str. Fuca to Farallone Is.

460 Rowellia radiata $C p$.

Farallone Is. to Catalina I.

461 Fissurella volcano live.

Santa Cruz to San Diego. Cai. Dd. Mrti. S muniguel

462 Fissurella violacea Esch.

Crescent City, Cal., to Sitka? S. Amer.

463 Glyphis aspera Esch.

Str. Fuca to Santa Barbara-Sitka. Cat. Jd. mi quel

464 Glyphis densiclathrata live.

Baulines Bay to San Diego. $=463 \mathrm{var}$

465 Lucapina crenulata Shy. Montcrey to San Diego. Catahina onty. San miguel

466 Puncturella cucullata Gld.

Str. Fuca to Monterey.

V. 467 Puncturella galeata Gid.

Str. Fuca, W. T.

408 Puncturella Cooperi Cpr.

Catalina I. 40 to 120 fins.

469 Clypidella callomarginata $C_{p r}$.

Lobitos to San Diego.

470 Clypidella bimaculata Dall, $\left(3 / s_{3}\right)$

Farallone Is. to IBarbarn I. Jan mi mee

471 Emarginula bella Gabb.

Santa Cruz to Monterey.

472 Haliotis Cracherodii Leach

Farallone Is. to San Diego-Lover Cal. Carahina, San miguel- Whty.

473 Haliotis splendens live,

Catalina I. to San Diego-Lower Cal. Catalina,

474 Haliotis corrugata Gray. Santa Barbara to San Diego.

Catalina

475 Haliotis rufescens $S_{w}$.

Farallone Is. to San Nicolas I-Galapagos? Catalina, Sun miquel- Nuty.

470 Haliotis Kamtschatkana Jonas.

Str. Fuca to Monteres-Japan. Kenai. mti.

477 Phasianella compta Gid.

San Pedro to Mazatlan. Caraina

478 Phasianclla compta rar? pulloides

Santa Cruz to San Dícgo. [Cpr. Catahina S an miguel, Het.

479 Pomaulax undosus Wood.

Santa Barbara to Cape St. Lucas-Monterey? Catalina, San mignel. 
458. May he var. of A.mitia or young of Patella pallida, lld + Lamanoni, schrenck.

459. Normal form of what Midd. desc, as eryptotranchia concentrica, subg. of Lepelā. Siltea, cringa. z-30fins. inainsthe cooks Intit.

460. Sadinia reticulatā, Shy. Subg. is inaginary and will not stand.

464. The densidathrata of the = aspera jun. The densidatha-

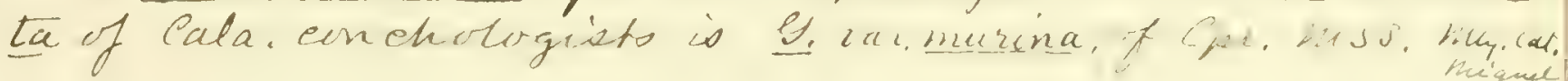

470. 2o probably an extreme var. of the preceding. - Fissurellidea not Clypidella. 

$4 \% 20$ Cyniscus, a. Ad, ano is Collonia Hrichii Crosse.

495. Add var. Fuctuatum tall. Mty. Catahina - San mignel-

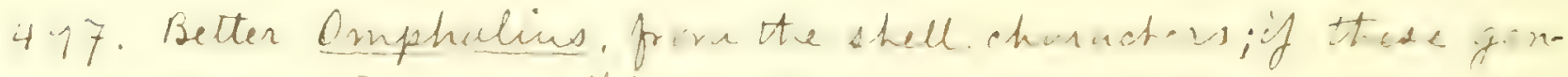
era are good for any thing.

499. Sitka?

500. A var. with trree the sutunal ribs, and another with allemate dark hount yethow radiating palches. mly. 
480 Pachypoma gibberosum Chem. Str. Fuca to Catalina I.- Nertratart?

481 Leptothyra sanguinea $C_{p} m$. Str. Fuca to San Diego-Japan-Europe! Mty. Catalina, rare

482 Leptothyra bacula $C_{p} r$. Santa Barbara to Catalina I.

catalina 2d-abrindant-lus I deep w.

483 Leptothyra paucicostata $D_{i l l},(1 / s s$. Monterey.

484 Liotia fenestrata $C_{p}$ r. Monterey to Catalina I. Mty. Catalina

485 Liotia acuticostata $C_{p}$. Monterey to Catalina I.

486 Ethalia supravallata $C p r$. San Diego-shell washings.

487 Ethalia invallata $C m$. San Diego-shell washings-var?

488 Livona picoides Gld. Pt. Conception or San Butenaventura? Cal.

489 Trochiscus Norrisii Sby. Santa Barbara to San Diego-Monterey I Wty. Catahina. San mu'guel.

490 Trochiscus convexus $C p r$. "Monterey," (Jewett coll.) Wiot a Trochis ens.

491 Chlorostoma funebrale $A . A d$. Str. Fuca to San Diego-Sitka. $m t_{4}$. Calalina sau Miguel.

492 Chlorostoma funebrale var. subaNeeah Bay, W. T. (Young?) [pertum Cpr.

493 Chlorostoma gallina Fbs. Cruz I., Cal., to Margarita Bay, Lower "Cal.

494 Chlorostoma gallina var. pyriformis San Diego.

[Gld.

495 Chlorostoma brunneum Phil. Catalina 2d- Nuty.

496 Chlorostoma Pfeifferi Phil. San Mignel 2d-Mh Baulines Bay to Nicolas I., Cal.

497 Chlorostoma aureotinctum $\mathrm{F}$ ls. Monterey? Santa Barbara to Lower Cal.

498 Omphalius fuscescens Phit. - Catalina I. to San Diego, Cal.

Catalina Id.

Catalina 2d-

499 Calliostoma canaliculatum Mart. Str. Fuca to Sau Diego, Cal.

500 Calliostoma costatum Mrart. Str. Fuca to Barbara I., Cal.-Sitka.

501 Calliostoma annulatum Mart. Str. Fuca to San Diego. ma. Caldinagd.

mas.

mty. Catalina 
502 Calliostoma variegatum Cpr.

Str. Fuca, W. T.

503 Calliostoma tricolor Gulb.

New Year loint to San Dicgo.

504 Calliostoma supragranosum $c_{j r}$

Santa Cruz to Montcrey.

505 Calliostoma gemmulatum $C_{p}$.

San Pedro to San Diego.

506 Calliostoma splendens $C_{\mu}$

Monterey to Cataliua I.

507 Thalotia caffea $(r, b)$

Ionterey, 20 fms. (S. Barb. and S. Pedro, F.)

508 Phorcus pulligo Mart.

Str. Fuca to Monterey-Sitka.

509 Gibbula parcipicta $C_{p}$.

Str. Fuca to Santa Barbara.

510 Gibbula optabilis $C_{p}$.

San Pedro, Cal.? (1, Palmer coll.)

511 Gibbula funiculata $C p r$.

Neeah Bay, W. T.

512 Gibbula succincta $C p$. Str. Fuca to Barbara I, Cal.

513 Gibbula lacunata $C_{p r}$. Neeah Bay, W. T.

514 Solariella peramabilis $C p r$. Catalina I., 40-1 $20 \mathrm{fms}$.

515 Margarita cidaris A. Ad. Necah Bay, W. T,

510 Margarita pupilla Gld. Str. Fuca to Monterey.

517 Margarita pupilla var. salmonea $C_{p r}$. Monterey to Catalina I.

518 Margarita acuticostata $C p r$. Baulines Bay to Santa Barbara.

2. 519 Margarita inflata $C p r$. Str. Fuca, IV. T.

520 Margarita lirulata $C p r$. Str. Fuca to Baulines Bay.

521 Margarita Vahlii 1foll. Str. Fuca, W. T.-Greenland.

522 Margarita tenuisculpta $C p r$. Str. Fuca, W. T.

U) 523 Margarita helicina Mont. Neeah Bay, W. T.-North Atlantic. Cook's mlet 


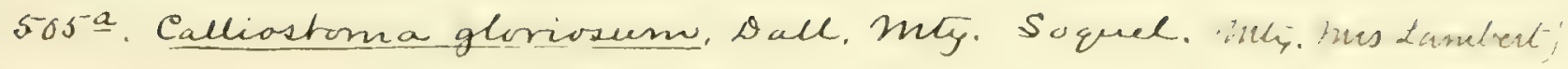
Add C. affinis. Sall, B. of Yeds. 2 apas.

506. Comp. Costatum jum.

506 add. C. Palmeri, Dall, Suaymad.

508. a . Iibbula Canfieldie, sall. Nty.

$509+510+511+512+513 ?+518+590+522=$ Mnargarita lisulata, vars.

$516+517+519=$ m. pupilla, vars.

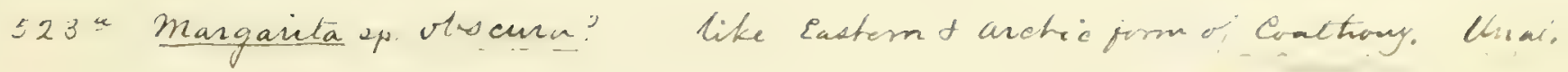

524 a Marqaila up. vorticifera, Wall, Hitaltened with sxpansed moutt, a food sutgenus. 



524 Galerus fastigiatus Gld.

Puget's Sound to Str. Fuca-Vancouver's I.

525 Galerus contortus $C p r$.

Lobitos Creek to Santa Barbara. Catalina 2d. Mhiy?

526 Crucibulum spinosum Sby.

San Pedro south to Peru, Sonth America. Monterey. Hanfond-Stearno.

527 Crucibulum scutellatum Gray.

Santa Cruz, Cal.-Lower Cal. to P'eru.

528 Crepidula aculeata Gmel.

Monterey south-Asia-Atlantic. Catalina

529 Crepidula dorsata Brod.

Str. Fuca to Santa Barbara? - Peru.

Calahina?

530 Crepidula dorsata var.? lingulata Gld. Str. Fuca to Mazatlan.

531 Crepidula excavata Brod.

Santa I3arbara? Sitka? Mexico to Peru.

532 Crepidula adunca Sby.

Str. Fuca to Santa Barbara-Nexico? San mignel - Catalena-

533 Crepidula rugosa $N u t t$.

Sta. Barbara to S. Diego-Mex, to Peru ?

534 Crepidula navicelloides Nutt. Str. Fuca to Santa Barbara.

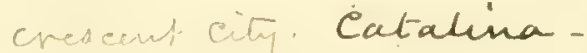

535 Crepidula navicelloides var. numStr. Fuca to San Pedro. [maria Gild.

536 Crepidula navicelloides var. fimbriStr. Fuca. [ata Rve.

537 Crepidula navicelloides var. explanStr. Fuca to San Pedro. [ata Gld.

538 Hipponyx cranioides $C p r$. Catahina

539 Hipponyx antiquatus Linn. Monterey south?-S. America. Atlantic. crescent lity cal rolus

540 Hipponyx serratus $C p r$. Santa Barbara south-Mazatlan.

541 Hipponyx tumens $C p r$. Monterey to San Diego and islands.

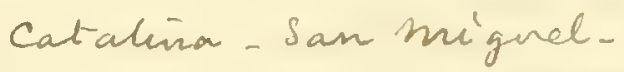

542 Serpulorbis squamigerus $C p r$. Santa Barbara to San Diego.

543 Bivonia compacta $C_{p r}$. Neeah Bay, W. T.

544 Petaloconchus macrophragma Cpr. San Diego to Panama.

545 Spiroglyphus lituella Morch.

- Santa Barbara to Cape St. Lucas. 
546 Cœeum crebricinctum $C p r$.

Monterey to San Diego.

547 Cœcum Cooperi $\mathrm{Cm}$.

San Diego Bay, 8-10 frns.

548 Turritella Cooperi $C_{p r}$.

Santa Barbara to San Diego.

549 Turritella Jewettii $C_{p}$ r.

Santa Barbara ; (Jewett coll., fossil ?)

550 Mesalia lacteola $\left.C_{p}\right)$ r.

l'uget's Sound to Vancouver's I.

551 Mesalia lacteola var.? subplanata $C_{p r}$ Str. Fuca, W. T.

552 Mesalia tenuisculpta $C_{p} p$. San Diego Bay, 4-6 fms., mul.

553 Cerithidea sacrata Gll. Baulines Bay to San Diego-Mazatlan?

554 Bittium filosum Gle. Str. Fuca to Monterey. Silka. Cookis Rulet.

555 Bittium filosum rar.? esuriens $C_{p} p$. Str. Huca to San Pedro.

556 Bittium attenuatum $C_{l} r$. Str. Fuca to Monterey.

557 Bittium quadrifilatum $C_{p r}$. Monterey to San Diego.

558 Bittium asperum $C_{p}$ r. Santa Barbara to Catalina I.

559 Bittium armillatum $C_{p}$ r. Furallone Is. to San Diego.

560 Bittium fastigiatum $C p$. Santa Barbara; (Jewett coll.)

561 Goniobasis occata IIds. Upper Sacramento River.

562 Goniobasis plicifera $L \epsilon a$. Willamette R., Oregon, to Montana.

563 Goniobasis rudens liee. Sacramento and Columbia Rivers.

564 Goniobasis silicula Glll. Shasta R., Cal., north, near coast.

565 Goniobasis Bairdiana Tea. "Near Astoria," Oregon. (Expl. Exp.)

566 Goniobasis Draytonii Ler. Napa and Butte Counties, Cal.-Oregon.

567 Goniobasis nigrina Lot. Napa Co. to Shasta Co., Cal. 
549. Add mesalia potaris, Beck, Aleutian 2slands, Plover Bay. $550=$ reticulata, mighels

$552 .=$ Mesalia acicula, ftrm in part, from type. 



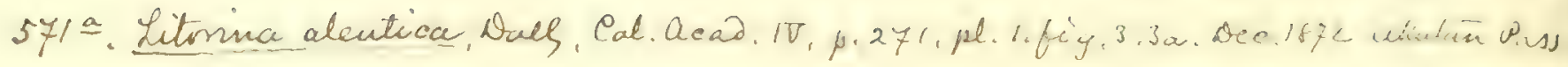


568 Goniobasis rubiginosa $L e a$.

"Oregon," Newcomb Mus., (Lea.)

569 Goniobasis circumlineata Tryon.

San Antonio Creek to Pit River, Cal.

570 Goniobasis Newberryi Lea.

Upper Des Chutes R., Uregon.

571 Goniobasis bulbosa Gld.

"Columbia R." (upper?) Expl. Exp.

572 Litorina planaxis Nutt.

Mendocino to San Diego-Sitka? Catalina - Nuy, Múguel.

573 Litorina Sitkana Phil.

Str. Fuca to Monterey-Sitka-Asia?

574 Litorina scutulata Gld.

Str. Fuca to San Diego.

Nyty. Catalina. Me'garel.

575 Assiminea? subrotundata $C p r$.

Neeah Bay, W. T,-Monterey.

576 Paludinella?

Neeah Bay, W. T.

577 Lacuna vincta Mont. Str. Fuca, W. T.--North Atlantic.

Tenai.

578 Lacuna porrecta $C \rho r$.

Neeah Bay, W. 'T., to Monterey.

579 Lacuna porrecta var? effusa $C p r$.

Neeah Bay, IV. T., to Lobitos, Cal.

580 Lacuna porrecta var. exæquata $C p r$.

S. F.? Neeah Bay, W. T.

581 Lacuna solidula Loven.

Str. Fuca to San Pedro-Norway.

582 Lacuna solidula var? compacta $C p r$.

Neeah Bay, W. T., to Monterey, Cal.

583 Lacuna variegata $C p r$.

Neeah Bay, IV. T.

584 Lacuna unifasciata $C p r$.

Farallone Is. to Santa Barbara

585 Iacuna unifasciata var. aurantiaca Catalina. Catalina I.

$[C p r$.

586 Isapis fenestrata $C p r$ -Str. Fuca to San Diego.

587 Isapis obtusa Cpr. Monterey to Sun Diego.

588 Rissoina interfossa $C p r$. Monterey to San Diego-deep water.

589 Rissoina purpurea $C p r$.

Monterey? Santa Barbara to San Diego. 6 
500 Rissoa compacta $C p r$. Str. Fuca, W. 'T.

591 Rissoa acutelirata Cpr. Santa Barbara to San Diego.

592 Rissoa? Cooperi Tryon. Monterey or San Diego. (Fresh water?)

593 Rissoa? exilis Tryon. Santa Barbara or S. Diego. (Fresh water?)

594 Alvania reticulata $C_{p}$. Necah Bay, W. T.

595 Alvania filosa $C_{Y r}$. Monterey, (from shell-washings.)

596 Fenella pupoidea $C_{p r}$. Monterey, 20 fms, dead.

597 Barleeia subtenuis $C p r$. Baulines? S. Diego-Mazatlan, Panama?

598 Barleeia subtenuis rar? rimata $C p r$. San Diego,

590 Barleeia haliotiphila $C p r$. Lower Cal.? ("On H. splendens," Ruvl.)

800 Amphithalamus inclusus $C p r$. Santa Barbara to San Diego.

BOI Amphithalamus? lacunatus $C P r$. San Pedro. (Palmer coll.)

602 Hydrobia? Californica Tryon. Oakland to Martinez, Cal.

603 Amnicola? turbiniformis Tryon. Northeast California, in streams.

604 Amnicola? longinqua Gld. Colorado Desert, (fossil only?)

605 Fluminicola Nuttalliana Lea. Willamette River, Oregon, near mouth.

600 Fluminicola fusca Hald. Sacramento R. to Green R., Utah.

607 Fluminicola Hindsii Baird. líotanie Kiver, Montana.

008 Fluminicola virens $L e a$. Willanette 1i., Oregon, near mouth.

BO9 Bythinella? intermedia Tryon. Owyhee Rircr, Southeast Oregon.

610 Bythinella Binneyi Tryon. Near Baulines Bay, Cal.

611 Cochliopa Rowellii Tryon. Clear Lake, California. 
595a. Alvania purpurea, Dale, My. 




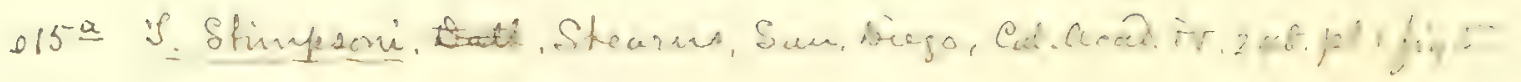


612 Tryonia clathrata Stp.

Colorado Desert, Cal. ; fossil only?

613 Tryonia protea Gld.

Colorado Desert, Cal.; fossil only?

614 Valvata virens Tryon.

Clear Lake, California.

615 Truncatella Californica $P f$.

San Yedro to San Diego-Mazatlan?

616 Jeffreysia Alderi $\mathrm{Cpr}$.

S. Diego-Cape St. Lucas to Mazatlan.

617 Jeffreysia translucens $C_{p}$ r.

S. Diego. (Burlecia or Rissoa?)

618 Cythna albida $C_{p}$.

San Pedro-Mazatlan? (=C. tumens Cpr ?)

619 Diala marmorea CPr.

Monterey to San Pedro-Cape St. Lucas. May. Calalicia.

620 Diala acuta $C p r$

Monterey to Catalina I.

621 Styliferina turrita $C p r$.

San Pedro; shell-washings. (Palmer coll.)

622 Radius variabilis $C . B . A d$.

Santa Barbara?-Lower Cal, to Panama.

623 Luponia spadicea $S w$.

Santa Barbara to San Diego and islands. Catatinn. Ne'guel-

624 Trivia Californiana Gray.

Monterey to San Diego and islands.

625 Trivia radians Lam.

S. Pedro-Lower Cal, to Peru.

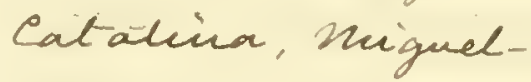

Calculina-

626 Trivia sanguinea Gray.

Catalina I. 60 f.-Lower Cal. to Peru.

627 Trivia Solandri Sby.

Santa Barbara and S. Nicolas I. to Panama.

628 Erato vitellina $H d s$.

Nerv Year Pt. to San Diego-Margarita Bay. Calahiea.

629 Erato columbella M1ke.

Monterey to San Diego-Mazatlan. Nety Cal-aliua

630 Terebra specillata $H d s$.

San Peuro-Cerros Is, to San Blas, L. Cal.

631 Myurella simplex $C p r$.

Santa Barbara to San Diego.

632 Drillia inermis IIrs.

Santa Barbara to San Diego-Lower Cal.

633 Drillia incisa $C p r$.

Str. Fuca, W. T., to Santa Cruz, Cal. 
634 Drillia mœsta $C_{p}$ r.

Santa Barbara to San Diego.

635 Drillia torosa $\mathrm{Cm}$.

Santa Cruz to Santa Burbara. M $\mathrm{Ny}_{4}$.

636 Drillia torosa $\alpha^{*}$ ? aurantia $C_{l}$ r.

San Pedro to Santa Barbara.

637 Drillia penicillata $C_{p r}$.

S. P'dro south-Cerros I. (=inermis vur?)

638 Drillia cancellata $C_{p}$ r.

Str. Fuca, W. T.

639 Sureula Carpenteriana Gelb.

Monterey to San Diego.

640 Pleurotoma perversa Gab.

Cataliua I. 60-120 fms., dead.

641 Mangelia levidensis $C_{\mu r}$. Str. Fuca, IV. T.

642 Mangelia tabulata $C_{l}{ }^{\prime}$. Neeah Bay, WV. T.

643 Mangelia interfossa $C p r$. Neeah Bay, W. T.

644 Mangelia crebricostata $C p$. Necah Bay, W. T.

645 Mangelia variegata $\mathrm{Cpr}$. Monterey to San Diego.

646 Mangelia variegata var? nitens $C p r$. Santa Barbara.

647 Mangelia angulata $C_{m}$. Str. Fuca to Santa Barbara.

648 Mangelia hexagona Galb. Monterey and Catalina I.

649 Clathurella

Catalina I. ; San Diego. (No. 663-3.)

650 Clathurella constricta Gubb. Catalina I. $80 \mathrm{f}$.

651 Clathurella crystallina Galb. Catalina I. 40 f.

652 Daphnella clathrata Gabb. Catalina I. $60 \mathrm{f}$.

653 Bela fidicula Gld. Str. Fuca, W. T.-Kamtschatka?

654 Bela excurvata Cpr. Str. Fuca, W. T.

655 Mitromorpha aspera $C p r$. Monterey, Cal. 
$633^{2}$ sillia montereyensis, stearns, Wh.

$633^{6}$ sillia Remphillie, Stearns, Lodos dantos Bay, L. Cal.

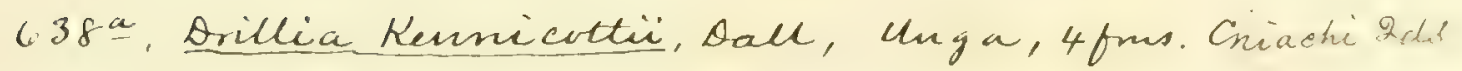

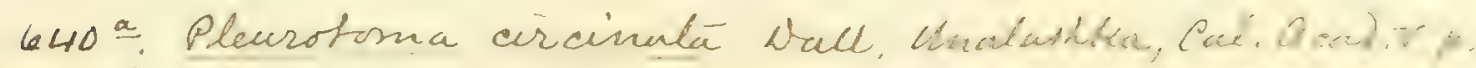

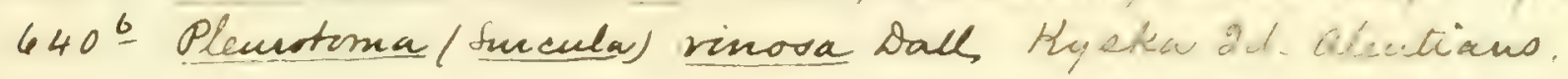

648‥ Mangelia alaskensis, Dall, Unga.

648 \% " alentica, Dall. Unga.

$648 \subseteq$ " funchale, Dwle, fithe.

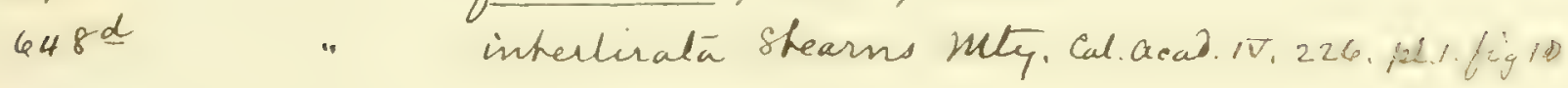

6519. Cathunella Cunfieldi, Dall, Mty.

657 . $\because$ affinis, bace, C.drtheas.

652. - Daphnella fuscoligatü, Dacl, Mty. Sandrigo, Jtäins

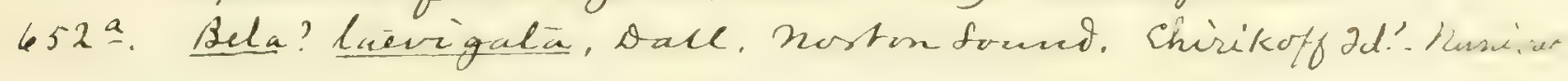




658 a Conns Dalti, Steans, Euct of bat.

$667^{a}$. Odostomia Beringi, Dall, noton formo. 
056 Mitromorpha filosa $C$ ro cataliua 2d, Lw.

657 Mitromorpha effusa $C_{p} m$. Neeah Bay, WV. T.

658 Conus Californicus Hinds. Farallone Is. to San Diegro-Lower Cal.

\section{Cutalina, Mty, San Nuguel.}

659 Obeliscus variegatus $\mathrm{Cpr}$.

San Diego-Cape St. Lucas; Guaymas.

680 Odostomia nuciformis $C_{p r}$.

Necah Bay, W. T.

661 Odostomia nuciformis var? avellana

Necah Bay, W. 'T.

662 Odostomia satura $C p r$.

Necah Bry, W. 'T:

663 Odostomia satura rar. Gouldii $C_{p} r$.

Neeah Bay, W. T.

664 Odostomia gravida Gild. Montercy to San Dizgo.

665 Odostomia inflata $C p$ r. Neeah Bay to Farallone Is.

666 Odostomia straminea Cpr. San Pedro to San Diego-Lower Cal.

667 Odostomia tenuisculpta $C_{p}$ r. Neeah Bay, W. T.

668 Chrysallida cincta $\mathrm{Cpr}$. Loc. uncertain, south of Monterey.

669 Chrysallida pumila $C_{p}$. Loc. uncertain, south of Monterey.

670 Dunkeria laminata $C_{p r}$. Santa Barbara to San Dicgo.

.671 Chemnitzia tridentata $C p r$. Str. Fuca to San Pedro.

672 Chemnitzia chocolata $C_{p r}$. Monterey to San Dicgo.

673 Chemnitzia chocolata var. aurantia Str, Fuca to Santa Burbara.

$\left[C_{l} r\right.$.

674 Chemnitzia tenuicula Gld. - Monterey to San Pedro.

675 Chemnitzia tenuicula var? subcuspiSan Diego, 6 fins. [data Cpr.

676 Chemnitzia crebrifilata $C_{p r}$. Santa Barbara.

677 Chemnitzia torquata Gld. Str. Fuca to Santa Barbara. 
675 Chemnitzia torquata $m$ stylina $C_{p}$. Monterey to Santa Barbara.

679 Chemuitzia virgo $C_{p} r$. Saner Marbara.

680 Chemuitzia Gabbiana $C_{p}$. Mt'y and Cat. I. ? (=gracillima Gabb not $C_{f} r_{0}$ )

681 Chemnitzia

Monterey to San Pedro. (No.459.)

652 Chemnitzia

Sau l)iego, dredged. (No. 428 a.)

683 Chemnitzia

Munterey to Catulina I. (No, 421.)

684 Chemnitzia

San Diego, dreclged (No. G7Ia.)

685 Eulima micans $C_{p} r$. Str. Fuca to Sun Diegu.

686 Eulima compacta $C_{p}$ r. San D) iego (=micuns var.?)

687 Éulima rutila $C_{M}$. Monterey (=micans $\iota^{2}$ ar.?)

688 Eulima thersites $\left.C_{p}\right)$. Monterey to Sitıta Barbara.

689 Scalaria Indianorum $C_{p r}$. Sir. Fuca to Bauliues; (and San Diego?)

690 Scalaria Indianorum var? tincta $C p r$. Santa Cruz to San Diego.

691 Scalaria Cumingii? Cpr. Sin Diego or islands? - l'anama.

692 Scalaria gracilis? Sby. Sin Diegro.

693 Scalaria subcoronata Cpr. Catalina $2 d$.

694 Scalaria creblicostata $C_{p}$ r. Montercy to San Dieno.

695 Scalaria bellastriata $C_{p}$ r. Sin Pedro to San Diego.

696 Opalia borealis Gld. Str. Fuca to San Pedro-Kamtschatka? Sfun ma a ta

697 Opalia crenatoides $C_{p} m$. Cape St. Lucas-l'ortugal?

698 Opalia crenatoides rar? insculpta Chr. Sinta Cruz to Santa Barbara. catalina 28

690 Opalia spongiosa $C_{p} p$. Monterey; shell washings, $\frac{1}{3}$ inch lons. 



708. Unga. Sitka. Unaiastha

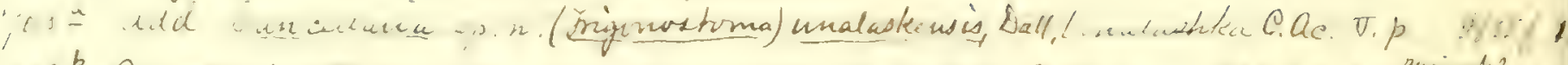

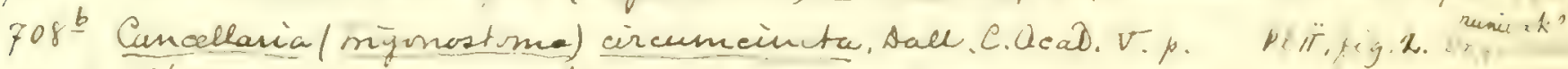
Foga Voluta (Bcaphella) Hearnsii, Dall, U-Sh. Cal. Uead. Tr. p. Lhe

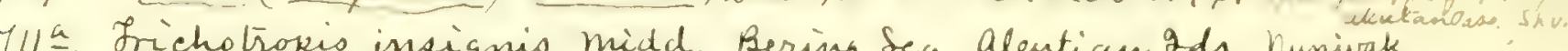
Ylla. Trichotropes insignis, midd. Bering Sea, Alentian 2ds, hunivak. Y11․ Fricholiopis hicarinatus, Shy. Bering Strait, Plover Bay. Nunivak $T_{11} \leqslant . \quad$ " " "var, allu, Dall. Plover Bay. Y11 " " " " "apectabilis, Drell, Seniavine Str. Yll E. 2phinöe peramabilis, Dall, Mnga.

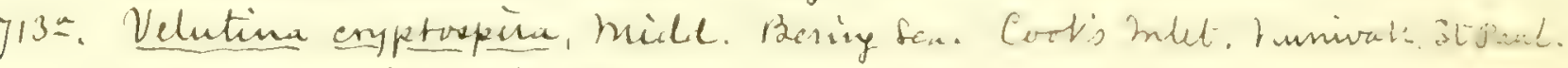

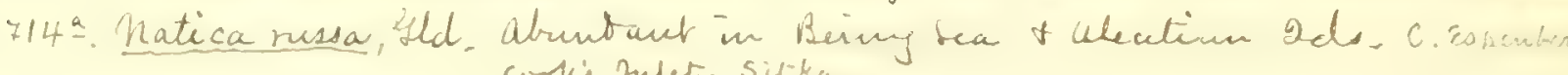
cookis metet. Sitka

717ㅇ. Amanvopsis purpuiren, Dall, Horton sound.

$719^{\circ}$ 2. Stearnsii, Dall.

720. L Shearnsii, var. vicutata, Dull.

721. L Numbica, Dace. 
700 Opalia retiporosa $C_{p} r$.

Catalina I. 40 fms.

701 Opalia bullata $C_{p}$ r.

Santa Barbara.

702 Cerithiopsis tuberculata Mont.

Str. Fuca to Catalina I.-Europe.

703 Cerithiopsis columna Crr. Sir. Fuca to San Diego.

704 Cerithiopsis munita $\mathrm{Cpr}$. Neeah Bay, W. 'T.

705 Cerithiopsis fortior $C p r$. Santa Barbara.

706 Cerithiopsis assimilata C.B. Ad. Monterey to Yanama.

707 Triforis adversa Mont. Str. Fuca to Catalina I.-England.

U. 708 Cancellaria modesta $C p r$. Neeah Bay, W. T.

709 Cancellaria Cooperi Gubu. Monterey to San Diego.

$L^{-} 710$ Trichotropis cancellata $H d s$ Str. Fuca and Puget Sound-Sitka.

i) 711 Trichotropis inermis Hds. Neeah Bay, W. T,-Sitka.

U. 712 Velutina lævigata Linn. Str. Fuca to Monterey-North Atlantic. Corolís nel"? Catalina?

713 Velutina prolongata $C p r$. Str. Fuca to New Ycar Point. Cerves Reter. Runimak?

714 Natica clausa Brod. \& Sby. Str. Fuca-Krmtschatka-North Atlantic. Kenou

715 Lunatia Lewisii Gld. Str. Fuca to San Diego. Mty.

U. 716 Lunatia pallida Brod. \&. Sby. Str. Fuea-Circumboreal.

717 Neverita Recluziana Petit. Monterey? Catalina I. to Lower Cal. Cat. Id-mly.

718 Sigaretus debilis Gld.

- Catalina 1. 40 f.-La Paz, Lower Cal. San Niguel.

719 Itamellaria stearnsii, ball Santa Cruz to Santa Barbara. (No. 1050.)

720 Lamellaria stearnsic, vac oriculata- dall Morterey.

721 Lamellaria thombica. Sael Montercy. 
V. 722 Priene Oregonensis Ticlf.

Str. Fuca to Monterey-Japan. Cookis Kulet.

723 Ranella Californica IIds. Monterey to San Diego.

eatakirea 2d

724 Mitra maura Sw.

Farallone I. to San Diego-South America? Cutaluira-luege-

725 Marginella Jewettii $C_{p}$.

Monterey to Santa Barbara.

726 Marginella subtrigona $C p r$.

Ilonterey" to Santa Barlara.

727 Marginella regularis $C_{p} r$.

Monterey to Sin Diego.

728 Marginella pyriformis $C_{p} r$. Monterey to San Diego.

729 Volvarina varia Sly. Santa Barbara to San Diego-Mexico; W. I. Catalina 20-Líy.

730 Olivella biplicata Sby. Str. Fuca to San Dicgo.

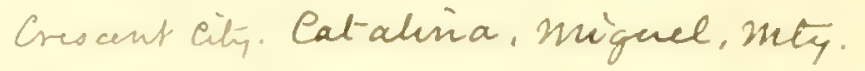

731 Olivella botica $C p r$. Str. Fuca to San Diego-Sitka.

Catalina 20.

732 Olivella dama Hnue. San I'edro-Gult' of Cal.

733 Olivella intorta $C m$. Baulines I3ay to Montercy; Gulf of Cal.?

734 Nassa fossata Cld. Sir. Fuca to San Diego.

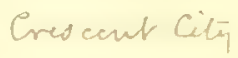

735 Nassa perpinguis Ilds. S. F. Bay to Sin Dicgo; Margarita Bay. Catalina 20.

736 Nassa insculpta $C_{p}$. Catalina I., California.

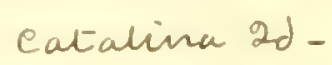

737 (Nassa mendica Gld. Str. Fuca to San Diegro.

738 Nassa Cooperi $\mathrm{k} / \mathrm{s}$. Baulines Bay to San Diego.

739 Nassa tegula live. Santa Barbara to San Diego-Lower Cal.

740 Amycla carinata $I I d s$. 1. Baulines Bay to Sin Diegro.

741 Amycla carinata var? Hindsii Rue. <Neeala Bay to San Diego.

742 Amycla gausapata Gld. Str. Fuca to San Diego.

743 Amyela gausapata rar? Californiana Sinta Cruz to San Diego. 


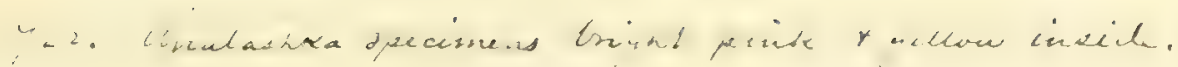

y23." ild Scaphella sp.(see Fog9) Uinalashke.

728. Persicula duhionas. Dall, Acapaled.

P.catenata, C.St. Lucas, Panuma, Sulapagos.

$1737=738$

740. 20 an astynis. $741+742+743$ are merely varietied. Cilalina inty Sim miguel. 


\section{.}



744 is an Dotyris.

$744^{\circ}$. Ade astyris auruntiaca, Aall, Hety. Catalina 28. 7446. Astynis variegata, shearm, dan diego.

746 . is not an Anycla.

$77^{\mathrm{a}}$, add Amphissa versievtov, Dall, mij. Suivmizuat.

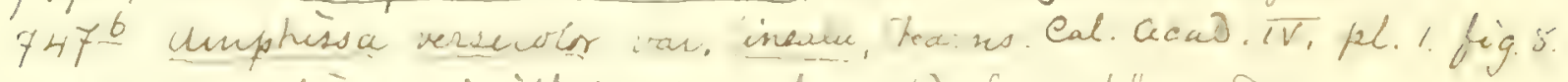
una his penieillata Che, Lun Pedo, dhepart.

758 a. Monoceros paucilirata stearns, Cormato 2S. Dan Diyo.

758 . Ocinelra gracillima mity. S. Diego.

758 - Ocinelra circumtexla

Cacialiusa 
744 Amyela tuberosa $C_{p}$ r.

Neealz Bay to San Diego.

745 Amycla chrysalloidea $C_{p} m$.

San Pedro and San Diegro. 746 Amycla undata $C r$ Catalina $2 d$.

747 Amphissa corrugata live.

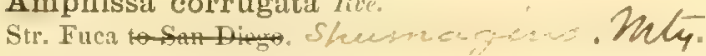

748 Purpura crispata Chem.

$\{$ Str. Fuca to Orcgon-Sitka. Crescent Cily- S. F. Bay-Mly, Kemai (smooth)

749 Purpura crispata $v$ restentrionalis

[live

U. 750 Purpura canaliculata Lucl.

Str. Fuca to Monterey-Beliring's Straits. Cresent Cily, Kenai- Catalena 2d. Mty.

751 Purpura saxicola $I^{\prime} a l$.

Str. Fuca to S. Barbara-Lower Cal.? Catalina 2d-

752 Purpura saxicola var. fuscata Fbs.

Str. Fuca to Santr Cruz, Cal.

753 Purpura saxicola vur. ostrina Gld. Str. Fucal to Santa Barbara.

754 Purpura saxicola var? emarginata S. F. Bay to S. 1)iego-N. Zcaland? [Desh.

755 Purpura triserialis Blainw.

Santa Barbara to San Diego-Mexico.

756 Monoceros engonatum Conr. Catalina 2d-Miguel - mly-

757 Monoceros engonatum var? spiratum

San Pedro to San Dicgo. [Blainv. 758 Monoceros lapilloides Conr. Caltahina 20 .
Monterey to Catalina I.

759 Ocinebra lurida Midel.

Str. Fuca to Catalina I. - Sitka.

760 Ocinebra lurida var. aspera Baird. Str. Fuca to Baulines Bay-Sitka.

761 Ocinebra lurida var. munda $C_{p}$ r. Str. Fuca to Baulines Bry.

762 Ocinebra lurida var? Santa Barbara to Catalina I.

763 Ocinebra interfossa $C p r$. Str. Fuca to Monterey. Curkis qulel

764 Ocinebra interfossa var. atropurStr. Fuca to Monterey. [purea $C_{p}$.

765 Ocinebra interfossa var. Santa Barbara to Catalina I. 
766 Ocinebra interfossa rur

Santa Cruz to San Diego.

707 Ocinebra Poulsoni Cpr.

Santa Barbara?-Cerros Is.; Panama!

768 Cerostoma foliatum Gurl.

Str. Fuca to Santa Barbara-Asia? Catalina 2d-

769 Cerostoma Nuttalii Conr.

Baulines Bay to San Dierro.

770 Cerostoma monoceros $s$

California? Lower Cal, and MIxico.

771 Chorus Belcheri IIds.

Catalina I. to San Diego; Lower Cal.

772 Nitidella Gouldii Cpr.

Str. Fuca to San Diego.

773 Pedicularia Californica Vcur.

Farallone Is, on coral, deep water.

774 Pteronotus festivus 1/rls. San 1'edro and San Diego-Lower Cal. Catalina Id

775 Muricidea incisa Brol. Cruz I. to Catalina I. Mexico; Panama. Catalina Id

776 Muricidea Californica $11 d s$. ? Baulines Iay to San Dicgo.

777 Muricidea Barbarensis Gabb. Santa Barbara to Catalina I.

778 Trophon multicostatus $\mathrm{Esch}$. Str. Fuca to Monterey-Sitka; Greenland. Cookis Zulet.

779 Trophon Orpheus Gild. Str. Fuca, IV. T.-Vancourer's I.

780 Trophon triangulatus $C_{p r}$. Cruz I. to Catalina I.

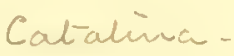

Catalma 75 tiwater-

781 Siphonalia Kelletii Fbs. Santa Barbara to San Diego-Japan.

782 Siphonalia fuscotincta $C p r$. Monterey to Catalina I.

783 Chrysodomus tabulatus $B d$. Str. Fuca to Catalina I.

784 Chrysodomus liratus Mart. Str. Fuca-Sitka; Kodiak I.

785 Chrysodomus dirus fire. Str. Fuca to Monterey-Sitka.

786 Chxysodomus rectirostris $C_{p}$ r. Str. Fuea, cast end, or P'uget's Sound.

787 Fusus ambustus Gld. Santa Barbara to Sin Diego-Mazatlan. 
7729. Add Nitidella elegans, Nall, Panama.

$77^{3} \cong$. "P.2aponica, Ball, 2apan.

774 ․ Jyphis sp.

Cutalina $2 d-$

774 - "Muricidea ep!

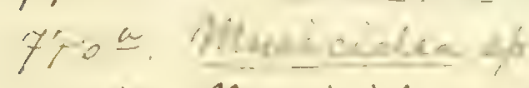

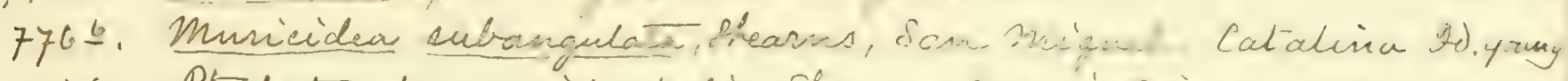

y7y. Plychatractus occidontalis, Shearns, Nayai. Onime.

784 , is quite distinct from decemcastaters, fay. E.C.

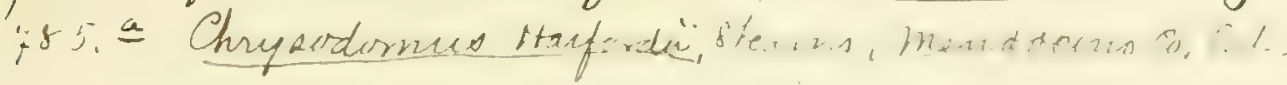

$7+6$.

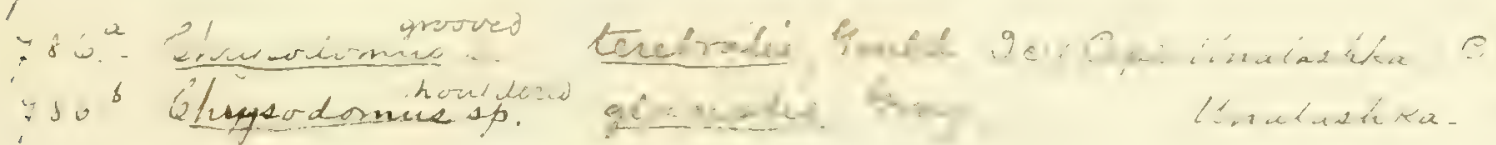

y87

786 c Cheyoodomes sp.

Kenai. 


$793=$ a. Pacifica. Aall. Catalina $2 d$.

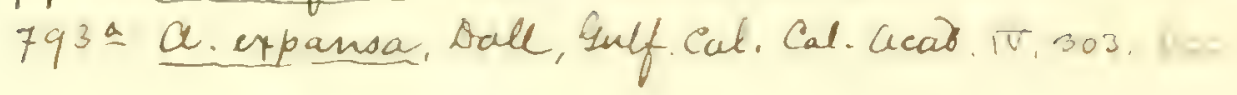

795 a. Onyehoteuthis Lobipensis. Aall, S.W. Farralones. 795 . Loliolus Steenstimpi, Dall. Lalf I Cala.

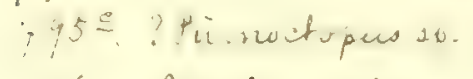

trinentitka.

$796=m \cdot$ Japomica 
788 Fusus geniculus Conr.?

Farallone Is. to Cruz I. (Oregon, foss.) Catalina $2 d$ -

789 Macron Kelletii $A$. $d d$.

California? Cerros Is, and Lower Cal.

790 Macron lividus A. Ad.

San Dicgo: (Farallone Is?)-Lower Cal. Catalina - abund ant.

791 Anachis subturrita $C p r$.

San Diego (shell washings.)

792 Anachis penicillata $C p r$.

Santa Barbara to San Diego.

793 Argonauta

Cruz I., south-Middle Atlantic.

794 Octopus punctatus Gabb.

San Francisco Bay to San Diego-Lower Cal.

795 Ommastrephes giganteus $D^{\prime} O r b$.

Clemente I., Cal._South America.

ADDITIONAL SPECIES FROM SITKA, ETC.*

796 Mya

Sitka to Kamtschatka-North Atlantic.

797 Astarte corrugata Brown.

Sitka-Circumpolar.

798 Placunanomia patellifermis tim.

Sitka?-Europe. = macho schied ona var:

799 不olis? crassicornis Esch.

Sitka.

800 死olis? subrosaceus Esch.

Sitka.

801 Eolidia pinnata Esch.

Sitka.

802 Dendronotus arborescens $1 / \mathrm{ull}$. Str. Fuca? Sitka-Circumpolar.

803 Planorbis Hornii Tryon.

Fort Simpson, lat. $54^{\circ} 40^{\circ}$.

804 Dentalium politum Linn.?

Sitka-North Atlantic.

- 805 Tonicia? insignis Rre.

Sitka.

806 Tonicia? Brandtii Midd.

Sitki.

807 Tonicia? Sitkensis Mild.

Sitkr.

* These species complete the list of known Mollusca of the temperate and boreal fauna of our coast, and many of them may yet be found south of lat. $49^{\circ}$. Others will doubtless prore identical with species of the frst list, or to bave been wrongly located on our coast. By adding to them all mentioned before as found either at Sitka or on each side of that place, the Mollusca of the locality may bo approximately known. It is likely that cevernl Arctic species also occur there, besides those mentioned in the first Jist, as there are no fixed limits to the regions, though pretty well separated by the peninsula of Aliaska. Some of the Lower Califoruia species, especially terrestrial, may be found to be limited to tlie temperate zone, but this is yet undecided. 
808 Tonicia? Escholtzii Midel. Sitka.

809 Lepidopleurus? lividus Mill. Sitka.

810 Lepidopleurus? scrobiculatus .Mill. Sitha? "California,"

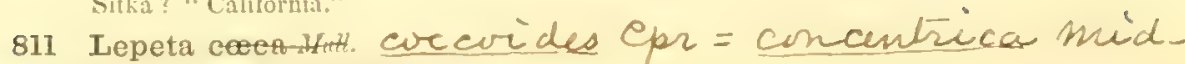

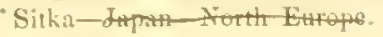

812 Haliotis aquatilis live. Sitka? -Japan.

813 Margarita aretica Leacte. Sitka; "var. major."

814 Crepidula grandis Nhidl.

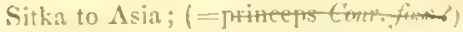

815 Lacuna glacialis 1 loll. Sitka-Circumpolar.

816 Litorina grandis Wikl.

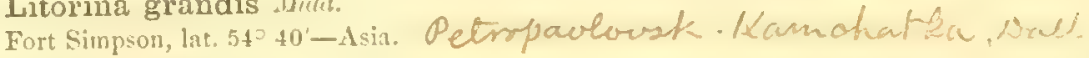

817 Velutina Sitkensis A. Al. Sitkr.

818 Chrysodomus tenebrosus Hun . Sitka-Lapland.

819 Chrysodomus carinatus Dink: Sittir.

820 Chrysodomus Morchianus Hero. .

821 Chrysodomus rutilus Mork. Sithr.

822 Chrysodomus Rombergi Dunk. itthert.

823 Chrysodomus harpa Moreh. Fitist Ley Cape, Mnal. Shemragins, Kadiak.

824 Chrysodomus castaneus Murch. sithis

825 Volutharpa ampullacea Midd. Sitka-Asia.

826. Buccinum ciliaterm, Jer Cape, C. Espenterg. NEW CALIFORNIAN SPECIRE.

163a Pisidium Angelicum liarl.

Angel I., Cal. (Rowell coll., Apr. 186i)

N. B.-l'ersons receiving two or more copies of this catalogne, will confer a faror by returaing one by mail to the State Geologist, with suclı notes as they may be able to add, for publication in the final report, where all such assistance will be daly ncknowledged. In conscquence of the time necesary for completing the engravings, the report carnot be issued in less than two years.

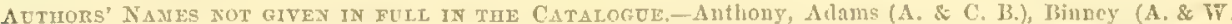

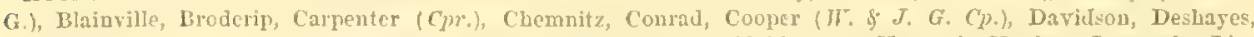
Dorbigny, Duclos, Dunker, Escholtz, Fabricins, Forbcs, Gmeliu, Halklemann, Irancock, Hanley, Lamarck, Lin-

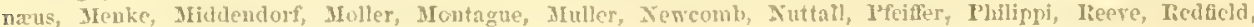
liowell, Sowerby, stimpson, Swainson, Valenciennes. 
811. This species has not heen found ittre in sitka or 2 apas.

813 a add margarita worticifera, ball, cal acen. Tip Mnalashka. Checitan Pas.

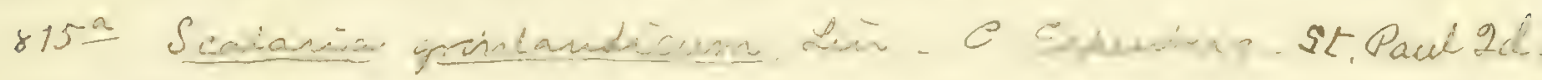

$818=$ Buccinumet.

$817^{\circ}$ add Buccinum talei sby wa related form, Nagai $W$.

$819=$ Buc. ylaciale. Lin. 2lin $+820+821+822+$ Hitimum angulrsum, Hoed all valo. Arctic to cork's Rulet. Icy Cape.

$823 .=$ Heliotropis harpan, hall. Cal. Aead..,$p \cdot 1873$. Shum-Unal.

$824 .=$ Volutopsis Beringi, midd. A.Ad. + Nept. radeá meverch t?

V. novegica, chems. Aleutians.? Krens.seas.

$824 a$ add Volulipsis var regulari Lall Cal. Acad. Tip. ili6

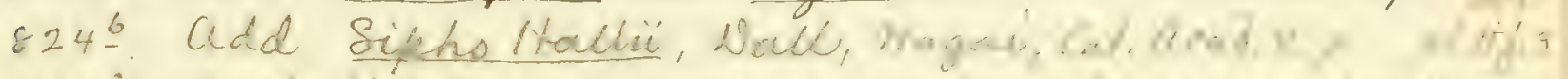

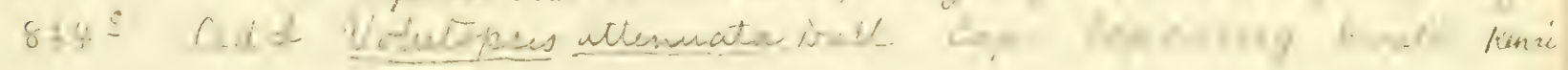

825. Add wax, acuminata. Bull, sether. Lypical at plover Bay and Detroparterok.

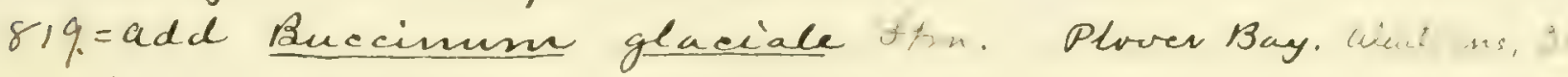

$819 \leqslant$

polare

8196 " cyaneum +Badi, sitkn, alenticuss. Minaí 819. Chrysodomus Remicotii. Nall, Shumagins 819 B Buccinum morchianum, Hischer, fitka 819 e .. Fischerianum, vall, ft. Leorge 28. 819 A Chrysodomes deforme Sray, Avalcha, Sharertiay.

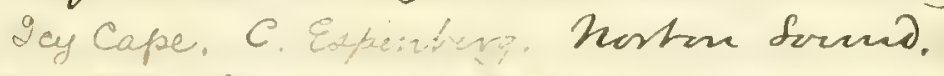

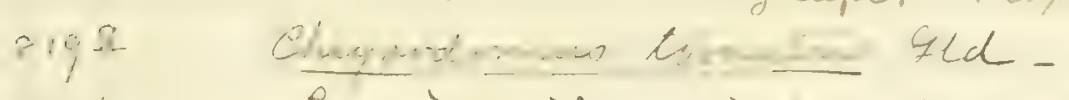

8.7- Buccinopois? canalicuiata Dall C. Espenberg, muminat. 


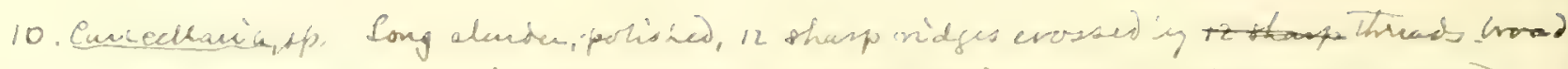
ardo frint, whols seven $1 \frac{2}{2} \operatorname{lon} g \times \frac{1}{2}$ wide. esinal shapply cenved

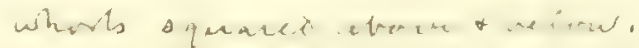

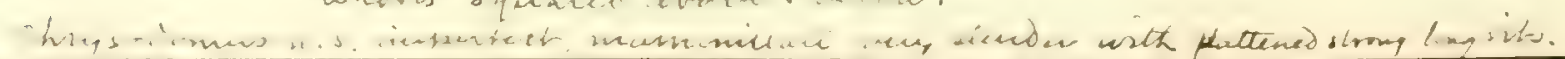


Pust pliocena fassils from Esmualda, Equaden. W.e.s.tm. collected ley Capk t. R. Hodgkins, Sche Unania

$$
1872-3
$$

Chama op - large.

Casts of Dosinia op-do.

Placunanomia macroschioma jun.

Ostrea sp - vey Large and heavy.

Pectewsp-like cuusina gled.

Arca grandis.

Jochita

Orucibuhum spinosum

. inbricatum

Crepidula, flat, coarsly diriale.

Pupura callar-ensis

Peripura (large ike melo)

Purpura vituberculata

Muricidea ep-esmall.

Comis

Cuma tectiformis = inskiformis.

Lurnitella tigrina

Jurbo (like pica)

Lilonina consper a.

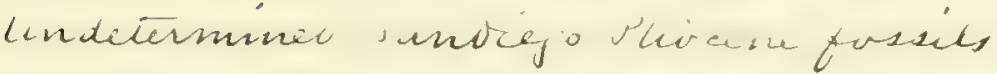

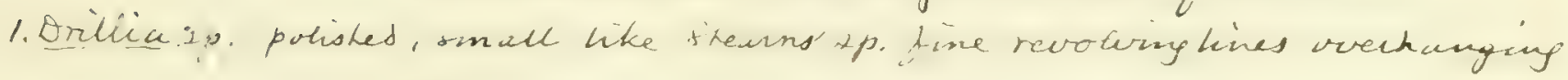
canal short.

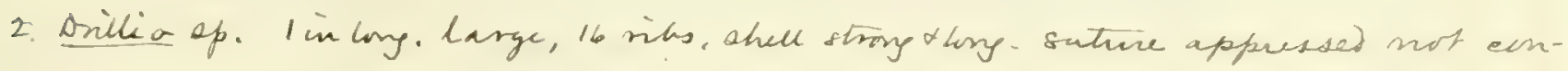
spicuoves, spiral senlptme, but evanescent on peripaces.

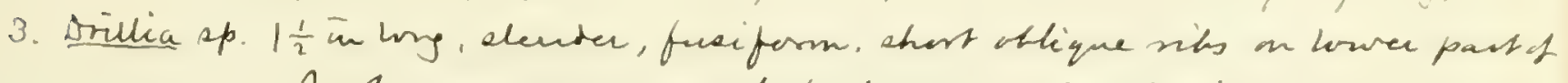

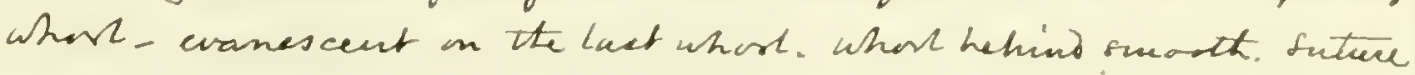

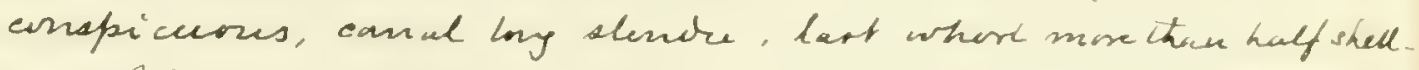

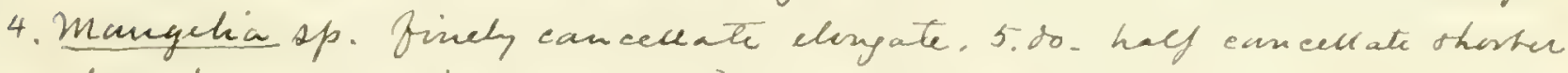
E. do - sheuply conoid bake wite, ohort thin canat.

7. Cancellaris. sp. Like M. Lapilivides, short, stout, merct usith fine revolving lines.

8. do tabulated, rattiue matium in length, sibs/f, atrongen than vevolering lines, fincly ecancellate.

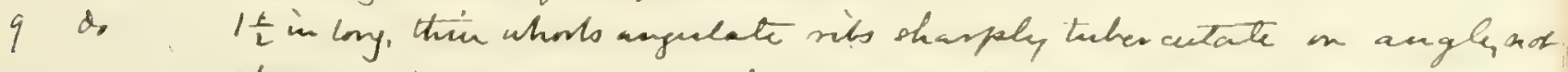

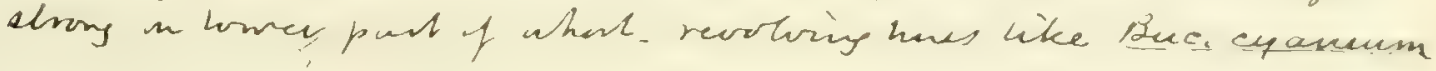





















Onried 


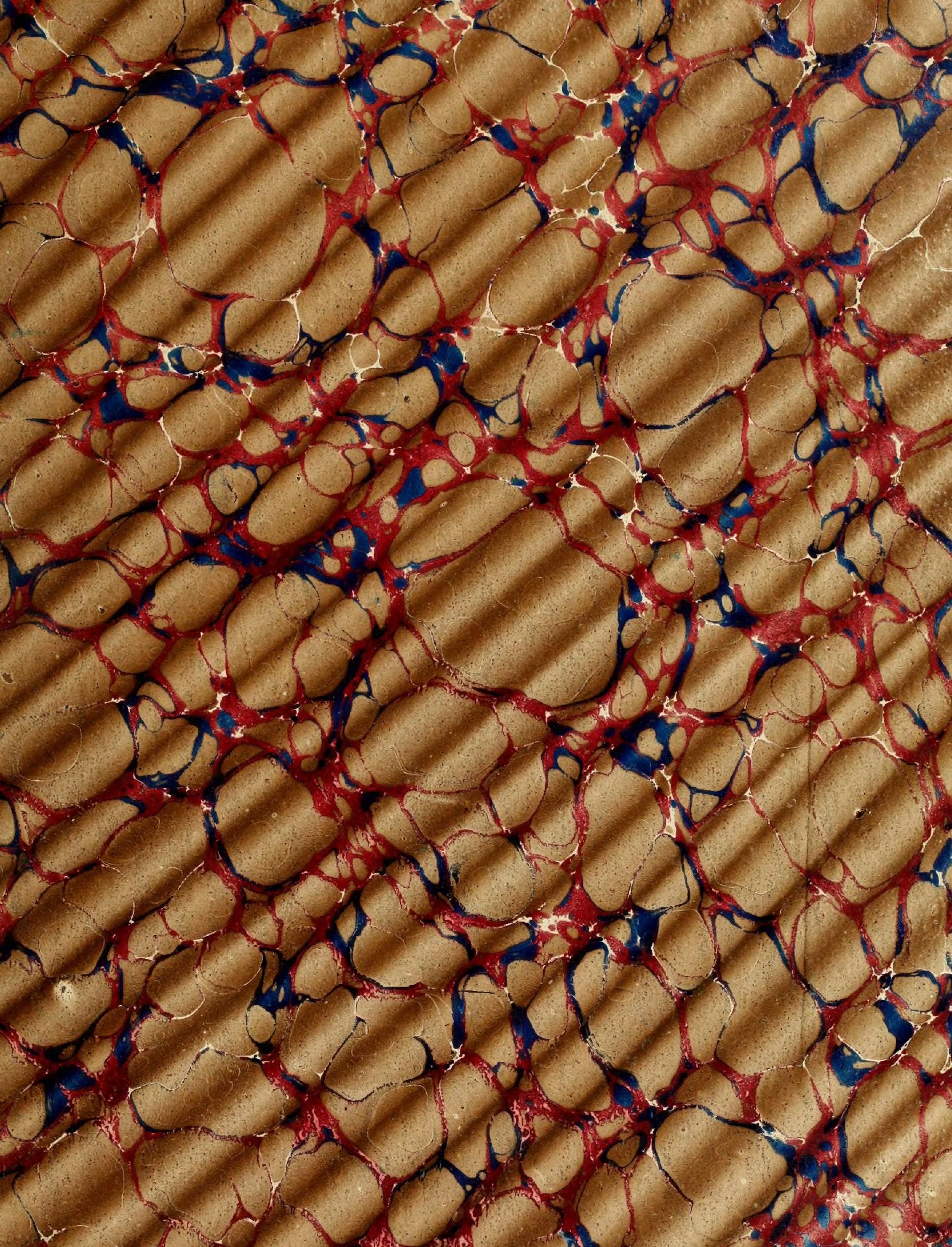

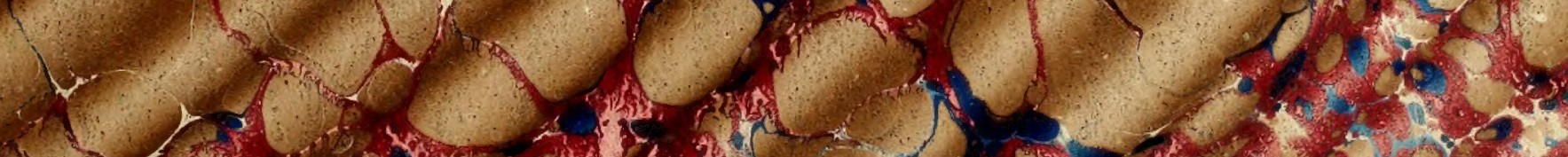
S.

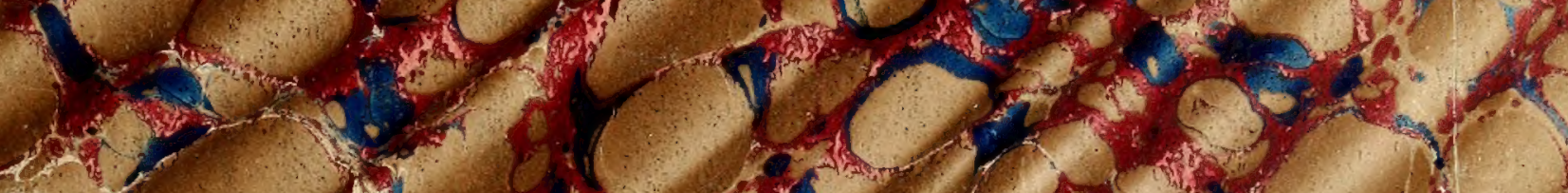

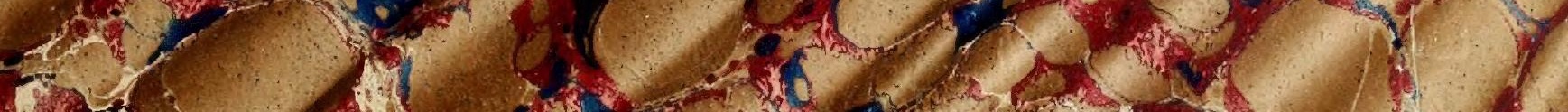


